\title{
BARGAINING AND EFFICIENCY IN NETWORKS
}

\author{
DILIP ABREU AND MIHAI MANEA
}

\author{
Department of Economics, Princeton University, dabreu@princeton.edu \\ Department of Economics, Harvard University, mmanea@fas.harvard.edu
}

\begin{abstract}
We study an infinite horizon game in which pairs of players connected in a network are randomly matched to bargain over a unit surplus. Players that reach agreement are removed from the network without replacement. For many networks all Markov perfect equilibria (MPEs) of the bargaining game are asymptotically inefficient as players become patient. The main result is that for every network structure the bargaining game admits asymptotically efficient subgame perfect equilibria. MPEs are of independent interest. We establish existence of MPEs, and show that MPE payoffs are not necessarily unique. We provide a method to construct pure strategy MPEs for high discount factors.
\end{abstract}

\section{INTRODUCTION}

In many markets buyers and sellers need to be in specific relationships in order to trade. A relationship may be defined by the possibility of production or assembly of a customized good (e.g., manufacturing inputs) or provision of a specialized service (e.g., technical support). Relationships may also encode transportation costs, social contacts, technological compatibility, joint business opportunities, free trade agreements, etc. In such markets transactions take place through the network of bilateral relationships. The structure of the network determines the nature of competition, the set of feasible agreements, and the potential gains from trade. In a recent book [11], Jackson surveys the emerging field of social and economic networks. He notes that the influence of the network structure on trading outcomes in noncooperative decentralized settings is a largely unexplored topic. The present paper and Manea (2008) attempt to fill this gap in two distinct strategic environments using non-cooperative models of decentralized bilateral bargaining in networks.

Date: May 11, 2009.

We are grateful to Drew Fudenberg for extensive discussions and feedback. We thank Fuhito Kojima and Al Roth for helpful comments. 
The setting is as follows. We consider a network where each pair of players connected by a link can jointly produce a unit surplus. The network generates the following infinite horizon discrete time bargaining game. Each period a link is randomly selected, and one of the two matched players is randomly chosen to make an offer to the other player specifying a division of the unit surplus between themselves. If the offer is accepted, the two players exit the game with the shares agreed on. If the offer is rejected, the two players remain in the game for the next period. In the next period the game is repeated on the subnetwork induced by the set of remaining players. We assume that all players have perfect information of all the events preceding any of their decision nodes in the game. All players have a common discount factor.

We can think of the model as a stylized account of the interaction between agents who have idiosyncratic supply and demand for some type of good or service. For instance, a particular contractor may have a process for sale (e.g., battery production) that only works for a subset of the firms in an industry (laptop manufacturing). Another contractor's process may only work for another subset and so on. In this setting one may ask: How are the relative strengths of the firms affected by the pattern of compatibilities (that is, the network structure)? Which partnerships are possible in equilibrium and on what terms? Is an efficient allocation of the processes achievable in equilibrium?

In our setting the production technology determines a maximum total surplus that may be generated cooperatively in every network. To achieve the maximum total surplus some pairs of connected players need to refrain from reaching agreements in various subgames. In general this requires that agreements arise only across specific "efficient" links, and that particular players be "saved" to trade with some players who might otherwise be isolated. For example, consider the 4-player network illustrated in Figure 1, which will be discussed in detail later. In this network efficiency requires that player 1 reach agreement with player 2, and player 3 with player 4, resulting in the maximum total surplus of two units. In particular, it is not efficient for player 2 to reach agreements with players 3 and 4 . However, for every discount factor the induced bargaining game has a unique Markov perfect equilibrium (MPE) in which agreement obtains with probability 1 across every link. Then with probability 1/2 one of the inefficient links $(2,3)$ and $(2,4)$ is selected for bargaining in the first period, and yields an agreement that leaves the other two players disconnected. In this event players do 


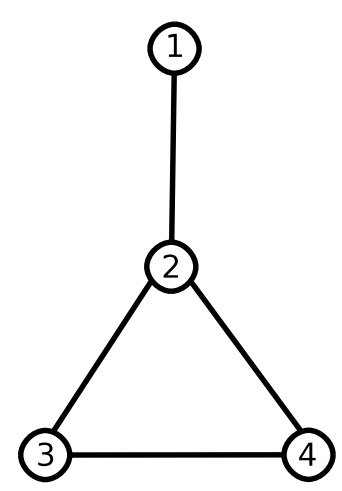

Figure 1. Asymptotically inefficient MPEs

not coordinate their agreements to generate a total surplus of two units, and only one unit of surplus is created on the equilibrium path.

Indeed, one can easily find networks for which no MPE of the bargaining game is efficient even asymptotically as players become patient (as in the example above). The main result of the paper is that for every network structure the bargaining game admits asymptotically efficient subgame perfect equilibria. Our equilibrium construction, of necessity, entails nonMarkovian behavior. Players who resist the temptation of reaching inefficient agreements are rewarded by their neighbors, and players who do not conform to the rewarding procedure are punished via the threat of inefficient agreements that result in their isolation.

Our result is not a direct adaptation of folk theorem type arguments. Note that the bargaining game is not a repeated game but rather a stochastic game. Folk theorem results for stochastic games have been established under the assumption that the feasible payoffs are independent of the initial state (Dutta 1995). This assumption is, of course, robustly violated in our setting, since agreement between a pair of players entails their irreversible removal from the network. Another distinction is that the stage game has an extensive form structure, but this distinction is perhaps less fundamental. ${ }^{1}$

In our setting it is non-trivial to explicitly specify any subgame perfect equilibrium, even for high discount factors. This contrasts with the usual folk theorem constructions. We establish existence of MPEs for the bargaining game via a fixed point argument rather than by explicit construction. The irreversible evolution of the network structure as play proceeds makes it difficult to check incentives. For that reason, our approach is to build as much as

\footnotetext{
${ }^{1}$ See Wen (2002) for an analysis of folk theorems for repeated sequential games.
} 
possible on an implicitly defined Markov strategy. As MPEs may be inefficient, we consider an MPE of a modified game which differs from the original game primarily in prohibiting inefficient agreements. This automatically accounts for most of the relevant incentives in the original game. The incentives to deviate that arise from the modifications of the original game are the only ones we need to address via explicit constructions of rewards and punishments.

Properties of MPEs are of independent interest. We prove that an MPE always exists, and demonstrate by example that MPE payoffs are not necessarily unique. We provide a method to construct pure strategy MPEs for high discount factors based on conjectures about the set of links across which agreement may obtain in every subnetwork.

Manea (2008) assumes that players who reach agreement are replaced by new players at the same positions in the network. The bargaining protocol is identical to the one of the present paper. The model is well-behaved in that equilibria are essentially unique and converge as players become patient. The main result is a procedure that determines the limit equilibrium payoffs by iterating the finding that players with extreme limit equilibrium payoffs form oligopoly subnetworks. An oligopoly subnetwork consists of a set of mutually estranged players and their bargaining partners; in equilibrium, for high discount factors, the partners act as an oligopoly for the mutually estranged players. In the limit, surplus within an oligopoly subnetwork is divided according to the shortage ratio of the mutually estranged players with respect to their partners. The main result is used to characterize equitable, stable, and non-discriminatory networks.

The two models differ in strategic complexity. In the model of Manea (2008) bargaining opportunities are stationary over time. A player's decisions consist solely in determining who his most favorable bargaining partners are. In effect, each player solves a search problem with prizes endogenously and simultaneously determined by the network structure. In the present model a player's decisions additionally entail anticipating that passing up bargaining opportunities may lead to agreements involving other players which undermine or enhance his position in the network in future bargaining encounters. Technically, this means that we need to compute equilibrium payoffs for every subnetwork that may arise following a series of agreements.

There is an extensive literature on bargaining in markets. Rubinstein and Wolinsky (1985, 1990), Gale (1987) and Binmore and Herrero (1988) consider markets where each seller owns 
one unit of an indivisible good, and each buyer demands one unit of the good. The focus is on the relationship between the equilibrium outcomes of various decentralized bargaining procedures and the competitive equilibrium price as the costs of search and delay become negligible. The findings are that the relationship is sensitive to the assumptions on the flow of agents entering the market over time, the amount of information available to agents, the matching technology, and the discount factor. All buyers and respectively all sellers are treated anonymously by the various stochastic matching processes considered in this literature. The analogue of this modeling assumption in our setting is the special case of buyer-seller networks in which every buyer is connected to every seller. For such networks, the payoffs in any MPE of our bargaining game converge to the competitive equilibrium outcome as players become patient. However, in our analysis the network is arbitrary. In particular, some pairs of buyers and sellers are not connected and cannot trade. Since bargaining encounters are restricted by network connections, the competitive equilibrium analysis does not apply.

Polanski (2007) studies a related model. The differences regard the matching technology and the steady state assumption. Polanski assumes that a maximum number of pairs of connected players are selected to bargain every period, and all players who reach agreement are removed from the network without replacement. Corominas-Bosch (2004) considers a model in which buyers and sellers alternate in making public offers that may be accepted by any of the responders connected to a specific proposer. As in the previous paper, the matching technology is chosen such that when there are multiple possibilities to match buyers and sellers (that is, there are multiple agents proposing or accepting identical prices) the maximum number of transactions take place. The efficient matching technologies of the preceding papers are fundamentally centralized. The market forces that would organize the matchings in order to maximize total surplus are not explicitly modeled by way of selfinterested strategic behavior.

Centralized trading mechanisms may be employed in order to implement efficient matching outcomes. Kranton and Minehart (2001) study a model similar to the one of CorominasBosch (2004). The valuations of the buyers are heterogeneous, and sellers are non-strategic. Prices are determined by the ascending-bid auction mechanism designed by Demange, Gale and Sotomayor (1986). The unique equilibrium in weakly undominated strategies leads to 
an efficient allocation of the goods. The result enables the study of the relationship between overall economic welfare and the incentives of buyers to form the network when links are costly to maintain.

Calvo-Armengol $(2001,2003)$ introduces the following model of sequential bargaining on a network. In the first round a proposer is randomly selected, and a responder is randomly chosen among his neighbors in the network. Every round that ends in disagreement is followed by a new round where the disagreeing responder makes an offer to a randomly chosen neighbor. The game ends when the first agreement is obtained. The unique stationary subgame perfect equilibrium specifies offers and responses identical to those in the two-person Rubinstein (1982) game. Therefore, the network has no effect on the ex-post equilibrium division, and only influences payoffs via the probabilities that an agent is the proposer or the responder in the first round of the game. These probabilities are defined exogenously in the model. Furthermore, it is assumed that a proposer passes down the bargaining opportunity to the responder in case of disagreement and that the game ends as soon as one pair reaches an agreement.

Formal models of non-cooperative two-player bargaining were introduced in the pioneering work of Stahl (1972), Rubinstein (1982) and Binmore (1987).

The rest of the paper is organized as follows. In Section 2 we define the model and establish existence of MPEs. Section 3 is concerned with asymptotic efficiency of equilibria, and Section 4 develops several properties of MPEs. The details of the proofs for Sections 2, 3, and 4 appear in Appendices A, B, and C, respectively. Section 5 concludes.

\section{FrameWORK}

Let $N$ denote the set of $n$ players, $N=\{1,2, \ldots, n\}$. A network is an undirected graph $H=(V, E)$ with set of vertices $V \subset N$ and set of edges (also called links) $E \subset$ $\{(i, j) \mid i \neq j \in V\}$ such that $(j, i) \in E$ whenever $(i, j) \in E$. We identify the links $(i, j)$ and $(j, i)$, and use the shorthand $i j$ or $j i$ instead. We say that player $i$ is connected in $H$ to player $j$ if $i j \in E$. We often abuse notation and write $i \in H$ for $i \in V$ and $i j \in H$ for

$i j \in E$. We denote by $e^{H}$ the total number of (undirected) links in $H$ and by $e_{i}^{H}$ the number of links player $i$ has in $H$. A player is isolated in $H$ if he has no links in $H$. A network $H^{\prime}=\left(V^{\prime}, E^{\prime}\right)$ is a subnetwork of $H$ if $V^{\prime} \subset V$ and $E^{\prime} \subset E$. A network $H^{\prime}=\left(V^{\prime}, E^{\prime}\right)$ is the 
subnetwork of $H$ induced by $V^{\prime}$ if $E^{\prime}=E \cap\left(V^{\prime} \times V^{\prime}\right)$. We write $H \ominus V^{\prime \prime}$ for the subnetwork of $H$ induced by the vertices in $V \backslash V^{\prime \prime}$.

Let $G$ be a fixed network with vertex set $N$. A link ij in $G$ is interpreted as the ability of players $i$ and $j$ to jointly generate a unit surplus. ${ }^{2}$ Consider the following infinite horizon bargaining game generated by the network $G$. Let $G_{0}=G$. Each period $t=0,1, \ldots$ a link $i j$ in $G_{t}$ is selected randomly (with equal probability), ${ }^{3}$ and one of the players (the proposer) $i$ and $j$ is chosen randomly (with equal probability) to make an offer to the other player (the responder) specifying a division of the unit surplus between themselves. If the responder accepts the offer, the two players exit the game with the shares agreed on. If the responder rejects the offer, the two players remain in the game for the next period. In period $t+1$ the game is repeated with the set of players from period $t$, except for $i$ and $j$ in case period $t$ ends in agreement, on the subnetwork $G_{t+1}$ induced by this set of players in $G$. Hence $G_{t+1}=G_{t} \ominus\{i, j\}$ if players $i$ and $j$ reach an agreement in period $t$, and $G_{t+1}=G_{t}$ otherwise. We assume that all players have perfect information of all the events preceding any of their decision nodes in the game. ${ }^{4}$ All players share a discount factor $\delta \in(0,1)$. The bargaining game is denoted $\Gamma^{\delta}(G)$.

There are three types of histories. We denote by $h_{t}$ a history of the game up to (not including) time $t$, which is a sequence of $t-1$ pairs of proposers and responders connected in $G$, with corresponding proposals and responses. We call such histories, and the subgames that follow them, complete. A complete history $h_{t}$ uniquely determines the set of players $N\left(h_{t}\right)$ remaining in the game at the beginning of period $t$; denote by $G\left(h_{t}\right)$ the subnetwork of $G$ induced by $N\left(h_{t}\right)$. Let $\mathcal{G}$ be the set of subnetworks of $G$ induced by the players remaining in any subgame, $\mathcal{G}=\cup_{h_{t}} G\left(h_{t}\right)$, and define $\mathcal{G}^{0}=\mathcal{G} \backslash\{G\}$. We denote by $\left(h_{t} ; i \rightarrow j\right)$ the history consisting of $h_{t}$ followed by nature selecting $i$ to propose to $j$. We denote by $\left(h_{t} ; i \rightarrow j ; x\right)$ the history consisting of $\left(h_{t} ; i \rightarrow j\right)$ followed by $i$ offering $x \in[0,1]$ to $j$.

A strategy $\sigma_{i}$ for player $i$ specifies, for all complete histories $h_{t}$ and all players $j$ such that $i j \in G\left(h_{t}\right)$, the offer $\sigma_{i}\left(h_{t} ; i \rightarrow j\right)$ that $i$ makes to $j$ after the history $\left(h_{t} ; i \rightarrow j\right)$, and the response $\sigma_{i}\left(h_{t} ; j \rightarrow i ; x\right)$ that $i$ gives to $j$ after the history $\left(h_{t} ; j \rightarrow i ; x\right)$. We allow for mixed strategies, hence $\sigma_{i}\left(h_{t} ; i \rightarrow j\right)$ and $\sigma_{i}\left(h_{t} ; j \rightarrow i ; x\right)$ are probability distributions

\footnotetext{
${ }^{2}$ We do not exclude networks in which some players are isolated.

${ }^{3}$ Our methods are not sensitive to the specification of the matching technology.

${ }^{4}$ The requirements on the information structure may be relaxed in the case of Markov perfect equilibria.
} 
over $[0,1]$ and $\left\{\right.$ Yes, No\}, respectively. A strategy profile $\left(\sigma_{i}\right)_{i \in N}$ is a subgame perfect equilibrium of $\Gamma^{\delta}$ if it induces Nash equilibria in subgames following every history $\left(h_{t} ; i \rightarrow j\right)$ and $\left(h_{t} ; i \rightarrow j ; x\right)$.

The equilibrium analysis is simplified if we assume that the only feature of a complete history of past bargaining encounters that is relevant for future behavior is the network induced by the remaining players following that history. That is, for all complete histories $h_{t}$ and all links $i j \in G\left(h_{t}\right)$, the offer $\sigma_{i}\left(h_{t} ; i \rightarrow j\right)$ that $i$ makes to $j$ only depends on $G\left(h_{t}\right), i, j$, and the response $\sigma_{i}\left(h_{t} ; j \rightarrow i ; x\right)$ that $i$ gives when $j$ offers $x$ only depends on $G\left(h_{t}\right), i, j, x$. Formally, a Markov strategy profile $\left(\sigma_{i}\right)_{i \in N}$ satisfies the following conditions

$$
\begin{aligned}
& \sigma_{i}\left(h_{t} ; i \rightarrow j\right)=\sigma_{i}\left(h_{t^{\prime}}^{\prime} ; i \rightarrow j\right) \& \sigma_{i}\left(h_{t} ; j \rightarrow i ; x\right)=\sigma_{i}\left(h_{t^{\prime}}^{\prime} ; j \rightarrow i ; x\right), \\
& \forall x, h_{t}, h_{t^{\prime}}^{\prime}, \forall i j \in G\left(h_{t}\right)=G\left(h_{t^{\prime}}^{\prime}\right) .
\end{aligned}
$$

A Markov perfect equilibrium (MPE) is a subgame perfect equilibrium in Markov strategies. ${ }^{5}$ We first establish existence of MPEs.

Proposition 1. There exists a Markov perfect equilibrium of the bargaining game $\Gamma^{\delta}(G)$.

The proof appears in Appendix A. For a set of networks $\mathcal{H}$, a collection of Markov strategy profiles $(\sigma(H))_{H \in \mathcal{H}}$ for the respective games $\left(\Gamma^{\delta}(H)\right)_{H \in \mathcal{H}}$ is subgame consistent if for every pair of networks $H, H^{\prime} \in \mathcal{H}, \sigma(H)$ and $\sigma\left(H^{\prime}\right)$ induce the same behavior in any pair of identical subgames of $\Gamma^{\delta}(H)$ and $\Gamma^{\delta}\left(H^{\prime}\right)$. By definition, any $\operatorname{MPE} \sigma^{* \delta}(G)$ of $\Gamma^{\delta}(G)$ must belong to a subgame consistent collection of MPEs $\left(\sigma^{* \delta}(\tilde{G})\right)_{\tilde{G} \in \mathcal{G}}$ of the respective games $\left(\Gamma^{\delta}(\tilde{G})\right)_{\tilde{G} \in \mathcal{G}}$. We use a bootstrap approach and a fixed point argument to (implicitly) construct an MPE $\sigma^{* \delta}(G)$ of $\Gamma^{\delta}(G)$ based on a subgame consistent family of MPEs $\left(\sigma^{* \delta}(G \ominus\{i, j\})\right)_{i j \in G}$ for the bargaining games $\left(\Gamma^{\delta}(G \ominus\{i, j\})\right)_{i j \in G}$. Behavior under $\sigma^{* \delta}(G)$ is specified according to $\sigma^{* \delta}(G \ominus\{i, j\})$ in any subgame that induces the network $G \ominus\{i, j\}$. Hence if we append $\sigma^{* \delta}(G)$ to $\left(\sigma^{* \delta}(G \ominus\{i, j\})\right)_{i j \in G}$ we obtain a larger family of subgame consistent MPEs. The proof of Proposition 1 proceeds by induction on the number of vertices in $G$.

\footnotetext{
${ }^{5}$ In other accounts $([15],[16])$, the concepts defined here would be referred to as stationary Markov strategies and stationary Markov perfect equilibrium.
} 


\section{Asymptotically Efficient Equilibria}

Fix a network $\tilde{G}$. We introduce some concepts for the purpose of studying the welfare properties of subgame perfect equilibria of the bargaining game $\Gamma^{\delta}(\tilde{G})$ for high $\delta$. A match of $\tilde{G}$ is a subnetwork of $\tilde{G}$ in which every player has exactly one link. The maximum total surplus of $\tilde{G}$, denoted $\mu(\tilde{G})$, is the maximum number of links in a match of $\tilde{G}$. An efficient match of $\tilde{G}$, generically denoted by $\tilde{M}$, is a match with $\mu(\tilde{G})$ links. A link is $\tilde{G}$ efficient if it is included in an efficient match of $\tilde{G}$, and $\tilde{G}$-inefficient otherwise. A player is always efficiently matched in $\tilde{G}$ if he is included in every efficient match of $\tilde{G}$. One simple observation that we use in the equilibrium construction for the forthcoming Theorem 1 is that if $i j$ is a $\tilde{G}$-inefficient link then $i$ and $j$ are always efficiently matched in $\tilde{G}$. A set of players $\tilde{\tilde{N}}$ in $\tilde{G}$ is $\tilde{G}$-efficiently closed if for any $\tilde{G}$-efficient link $l m$, the set $\{l, m\}$ either is contained in $\tilde{\tilde{N}}$ or has empty intersection with $\tilde{\tilde{N}}$.

We measure the welfare of an equilibrium $\sigma^{* \delta}(\tilde{G})$ of $\Gamma^{\delta}(\tilde{G})$ as the sum of expected utilities of all players in that equilibrium, denoted $W\left(\sigma^{* \delta}(\tilde{G})\right)$. Since each player can only be involved in one transaction, each transaction yields a unit surplus, and only connected pairs of players can transact, for every $\delta \in(0,1)$ and any equilibrium $\sigma^{* \delta}(\tilde{G})$ of $\Gamma^{\delta}(\tilde{G})$ the welfare $W\left(\sigma^{* \delta}(\tilde{G})\right)$ cannot exceed $\mu(\tilde{G})$. For $\underline{\delta} \in(0,1)$, a family of equilibria $\left(\sigma^{* \delta}(\tilde{G})\right)_{\delta \in(\underline{\delta}, 1)}$ corresponding to the games $\left(\Gamma^{\delta}(\tilde{G})\right)_{\delta \in(\underline{\delta}, 1)}$ is asymptotically efficient if $\lim _{\delta \rightarrow 1} W\left(\sigma^{* \delta}(\tilde{G})\right)=\mu(\tilde{G})$.

To generate the maximum total surplus $\mu(\tilde{G})$ in $\Gamma^{\delta}(\tilde{G})$ as players become patient, pairs of players connected by links that are inefficient in the induced subnetworks in various subgames need to refrain from reaching agreements. However, providing incentives against agreements that are collectively inefficient is a difficult task. The difficulty springs from the anxiety of some players that passing up bargaining opportunities may lead to agreements involving their potential bargaining partners which undermine their position in the network in future bargaining encounters. Indeed, one can easily find networks for which all MPEs of the bargaining game are asymptotically inefficient as players become patient.

The network $G_{t r+p o i n t}$ illustrated in Figure 2 induces the simplest bargaining game that does not posses asymptotically efficient MPEs. For every $\delta \in(0,1)$, in the unique MPE of $\Gamma^{\delta}\left(G_{t r+p o i n t}\right)$ agreement occurs with probability 1 across every link in the first period. Proposition 4 from the next section supplies the limit MPE payoffs: $11 / 56 \approx .196$ for 


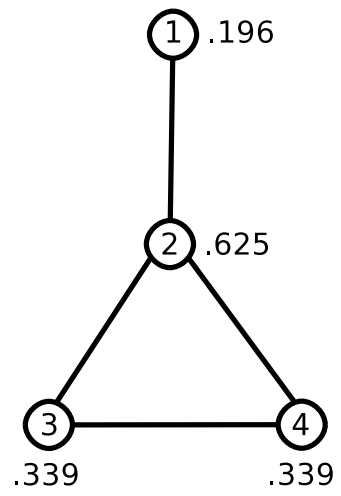

Figure 2. Asymptotically inefficient MPEs for the bargaining game on $G_{t r+\text { point }}$

player $1,5 / 8=.625$ for player 2 , and $19 / 56 \approx .339$ for players 3 and 4 . The limit MPE welfare is $11 / 56+5 / 8+2 \times 19 / 56=3 / 2$, which is smaller than the maximum total surplus $\mu\left(G_{t r+\text { point }}\right)=2$. The set of MPEs is not asymptotically efficient because, for every $\delta \in(0,1)$, in the unique MPE of $\Gamma^{\delta}\left(G_{t r+\text { point }}\right)$, with probability $1 / 2$ one of the $\Gamma^{\delta}\left(G_{t r+\text { point }}\right)$-inefficient links $(2,3)$ and $(2,4)$ is selected for bargaining in the first period, leading to an agreement that leaves the other two players disconnected. In this event players do not coordinate their agreements in order to generate the maximum total surplus of two units, and only one unit of surplus is created on the equilibrium path.

We seek to show that for every network structure $G$ the bargaining game $\Gamma^{\delta}(G)$ admits asymptotically efficient equilibria. For this purpose, we introduce a new bargaining game, $\bar{\Gamma}^{\delta}(G)$, which is a modification of the benchmark bargaining game $\Gamma^{\delta}(G)$ that "prohibits" equilibrium agreements across inefficient links in any subgame by adding a fine of -1 to the regular payoffs of any player involved in such an agreement. In addition, for particular subnetworks that may be induced by subgames, if certain efficient links and proposers are chosen, $\bar{\Gamma}^{\delta}(G)$ "imposes" equilibrium agreements via fining the proposer with a payoff of -1 in case his offer is rejected. In all other respects $\bar{\Gamma}^{\delta}(G)$ is identical to $\Gamma^{\delta}(G)$. It is clear that the artificial payoff modifications induce the desired disagreements and agreements in any equilibrium of $\bar{\Gamma}^{\delta}(G)$. The key idea is to employ MPE payoffs of $\bar{\Gamma}^{\delta}(G)$ in the construction of non-Markovian asymptotically efficient equilibria for $\Gamma^{\delta}(G)$ based on rewards and punishments.

The following concepts are necessary for the definition of $\bar{\Gamma}^{\delta}(G)$ (and the rest of the equilibrium construction). A network $\tilde{G}$ is perfect if in $\tilde{G}$ all non-isolated players are 
always efficiently matched. A network $\tilde{G}$ is non-trivial perfect if $\tilde{G}$ is perfect and there exists a $\tilde{G}$-inefficient link. A quadruple of players $h-i-j-k$ forms a core configuration in $\tilde{G}$ if $i j$ is $\tilde{G}$-inefficient and there is an efficient match of $\tilde{G}$ that includes the links $i h$ and $j k$. Note that every non-trivial perfect network $\tilde{G}$ must contain a core configuration. ${ }^{6}$ For each such network $\tilde{G}$ we fix a core configuration $h(\tilde{G})-i(\tilde{G})-j(\tilde{G})-k(\tilde{G})$. The functions $h(\cdot), i(\cdot), j(\cdot), k(\cdot)$ are defined for all non-trivial perfect networks and are part of the description of $\bar{\Gamma}^{\delta}(G)$.

We can now formally define the modified bargaining game $\bar{\Gamma}^{\delta}(G)$ generated by the network $G$. Let $G_{0}=G$. Each period $t=0,1, \ldots$ a link $i j$ in $G_{t}$ is selected randomly (with equal probability), and one of the players (the proposer) $i$ and $j$ is chosen randomly (with equal probability) to make an offer to the other player (the responder) specifying a division of the unit surplus between themselves. If the responder accepts the offer, the two players exit the game (1) with the shares agreed on if $i j$ is $G_{t}$-efficient, and (2) with the shares agreed on minus 1 if $i j$ is $G_{t}$-inefficient. If the responder rejects the offer, the two players remain in the game for the next period; if $G_{t}$ is non-trivial perfect, and the proposer and responder are $k\left(G_{t}\right)$ and respectively $j\left(G_{t}\right)\left(h\left(G_{t}\right)\right.$ and respectively $\left.i\left(G_{t}\right)\right)$, then $k\left(G_{t}\right)\left(h\left(G_{t}\right)\right)$ receives a time $t$ payoff of $-1 .^{7}$ At time $t+1$ the game is repeated with the set of players from time $t$, except for $i$ and $j$ in case period $t$ ends in agreement, on the subnetwork $G_{t+1}$ of $G$ induced by this set of players. Hence $G_{t+1}=G_{t} \ominus\{i, j\}$ if players $i$ and $j$ reach an agreement at time $t$, and $G_{t+1}=G_{t}$ otherwise. All players have perfect information of all the events preceding any of their decision nodes in the game and share the discount factor $\delta \in(0,1)$.

As in the case of the benchmark bargaining game $\Gamma^{\delta}(G)$, a Markov perfect equilibrium for the modified bargaining game $\bar{\Gamma}^{\delta}(G)$ is defined as a subgame perfect equilibrium in strategies that only condition on each complete history of past bargaining encounters through the network induced by the remaining players after that history. The formal definition can be easily inferred from the case of $\Gamma^{\delta}(G)$, and is omitted. Trivial modifications to the proof of Proposition 1 show existence of MPEs for $\bar{\Gamma}^{\delta}(G)$.

\footnotetext{
${ }^{6}$ If $i j$ is $\tilde{G}$-inefficient then $i$ and $j$ are always efficiently matched in $\tilde{G}$. Let $h$ and $k$ be the neighbors of $i$ and respectively $j$ in an arbitrary efficient match of $\tilde{G}$. Then the quadruple $h-i-j-k$ forms a core configuration in $\tilde{G}$.

${ }^{7}$ With this payoff modification some players may receive non-zero payoffs in more than one period. However, this cannot happen in equilibrium.
} 
Proposition 2. There exists a Markov perfect equilibrium of the modified bargaining game $\bar{\Gamma}^{\delta}(G)$.

For each $\delta \in(0,1)$, fix an MPE $\bar{\sigma}^{* \delta}(G)$ of $\bar{\Gamma}^{\delta}(G)$. Due to the artificial payoff modifications defining $\bar{\Gamma}^{\delta}(G)$, it must be that under $\bar{\sigma}^{* \delta}(G)$, in any subgame that induces the network $\tilde{G}$, with probability 1 , disagreement obtains across $\tilde{G}$-inefficient links, and agreement obtains when players $h(\tilde{G})$ and $k(\tilde{G})$ are selected to propose to $i(\tilde{G})$ and respectively $j(\tilde{G})$ (if $\tilde{G}$ is non-trivial perfect). In this sense $\bar{\sigma}^{* \delta}(G)$ "prohibits" agreements in the former situations and "imposes" agreements in the latter. The following definitions apply for all subnetworks $\tilde{G} \in \mathcal{G}$. Let $\left(\bar{\sigma}_{i}^{* \delta}(\tilde{G})\right)_{i \in \tilde{G}}$ be the MPE of $\bar{\Gamma}^{\delta}(\tilde{G})$ determined by $\bar{\sigma}^{* \delta}(G)$ in a subgame of $\bar{\Gamma}^{\delta}(G)$ where the remaining players induce the network $\tilde{G}$, and $\left(v_{i}^{* \delta}(\tilde{G})\right)_{i \in \tilde{G}}$ be the payoffs yielded by $\bar{\sigma}^{* \delta}(\tilde{G})$, also called the $\tilde{G}$-quasi-Markov payoffs. Denote by $p^{* \delta}(\tilde{G} ; l m)$ the first period agreement probability across the link $l m \in \tilde{G}$ in $\bar{\Gamma}^{\delta}(\tilde{G})$ under $\bar{\sigma}^{* \delta}(\tilde{G})$ (conditional on $l$ and $m$ being matched for bargaining).

As mentioned already, the idea is to employ the quasi-Markov payoffs in the construction of asymptotically efficient equilibria of $\Gamma^{\delta}(G)$. Strategies in the constructed equilibrium determine a distribution over bargaining outcomes in $\Gamma^{\delta}(G)$ identical to the distribution induced by the MPE $\bar{\sigma}^{* \delta}(G)$ in $\bar{\Gamma}^{\delta}(G)$, and yield payoffs equal to the $G$-quasi-Markov payoffs $v^{* \delta}(G)$. Incentives to deviate are offset by rewards and punishments. Players who resist the temptation of reaching inefficient agreements are rewarded by their neighbors. Players who do not conform to the rewarding procedure, and players $h(\tilde{G})$ and $k(\tilde{G})$ whose offers are rejected by $i(\tilde{G})$ and respectively $j(\tilde{G})$ in subgames with induced (non-trivial perfect) subnetwork $\tilde{G}$ are punished via the threat of inefficient agreements that result in their isolation. The reference points for rewards and punishments are (typically) the quasi-Markov payoffs for the corresponding subgames. Definition 1, Lemmata 1-5 and Proposition 3 below, concerning limit equilibrium agreements and payoffs in various subgames of $\bar{\Gamma}^{\delta}(G)$ under $\bar{\sigma}^{* \delta}(G)$, are necessary for constructing the equilibrium strategies and verifying the incentives.

Definition 1. A sequence of discount factors $\left(\delta_{\alpha}\right)_{\alpha \geq 0}$ with $\lim _{\alpha \rightarrow \infty} \delta_{\alpha}=1$ is well-behaved if the sequences $\left(v_{g}^{* \delta_{\alpha}}(\tilde{G})\right)_{\alpha \geq 0}$ and $\left(p^{* \delta_{\alpha}}(\tilde{G} ; i j)\right)_{\alpha \geq 0}$ converge as $\alpha \rightarrow \infty$ for every $g \in \tilde{G}$ and respectively every $i j \in \tilde{G}$, for all $\tilde{G} \in \mathcal{G}$. 
A sequence of discount factors converging to 1 is well-behaved if the payoffs and agreement probabilities under $\bar{\sigma}^{* \delta}(G)$ across all subgames of $\bar{\Gamma}^{\delta}(G)$ converge for $\delta$ along the sequence. The statements of Lemmata 1-5 and Proposition 3 apply for each (fixed) subnetwork $\tilde{G} \in \mathcal{G}$, and every (fixed) well-behaved sequence of discount factors $\left(\delta_{\alpha}\right)_{\alpha \geq 0}$. The corresponding limits of $\left(v_{g}^{* \delta_{\alpha}}(\tilde{G})\right)_{\alpha \geq 0}$ and $\left(p^{* \delta_{\alpha}}(\tilde{G} ; i j)\right)_{\alpha \geq 0}$ as $\alpha \rightarrow \infty$ are denoted by $v_{g}^{*}(\tilde{G})$ and $p^{*}(\tilde{G} ; i j)$, respectively. The proofs appear in Appendix B.

Lemma 1. Let $\tilde{\tilde{N}}$ be a $\tilde{G}$-efficiently closed set of players that induces the subnetwork $\tilde{\tilde{G}}$ in $\tilde{G}$. Suppose that $\tilde{\tilde{G}}$ has a non-empty set of links, and let $\tilde{\tilde{M}}$ be an efficient match of $\tilde{\tilde{G}}$. Then there exists a link $l m \in \tilde{\tilde{M}}$ such that $p^{*}(\tilde{G} ; l m)=1$.

We obtain the corollary below by setting $\tilde{\tilde{N}}$ equal to the set of vertices of $\tilde{G}$ in Lemma 1.

Corollary 1. Suppose that $\tilde{G}$ is a network with a non-empty set of links, and let $\tilde{M}$ be an efficient match of $\tilde{G}$. Then there exists a link $l m \in \tilde{M}$ such that $p^{*}(\tilde{G} ; l m)=1$.

Remark 1. One consequence of Corollary 1 is that

$$
\lim _{\delta \rightarrow 1} \sum_{i \in N} v_{i}^{* \delta}(G)=\mu(G)
$$

Thus a family of equilibria (whose existence we intend to establish) of $\Gamma^{\delta}(G)$ yielding the payoffs $v^{* \delta}(G)$ for sufficiently high $\delta$ must be asymptotically efficient.

The next result establishes that players always efficiently matched in $\tilde{G}$ are relatively strong in $\bar{\Gamma}^{\delta}(\tilde{G})$, in the sense that their limit $\tilde{G}$-quasi-Markov payoffs are greater than or equal to $1 / 2$.

Proposition 3. Suppose that player $i$ is always efficiently matched in $\tilde{G}$. Then $v_{i}^{*}(\tilde{G}) \geq 1 / 2$. If additionally $\tilde{G}$ is a perfect network, then $v_{i}^{*}(\tilde{G})=1 / 2$.

The following two lemmata are used in designing reward paths in the construction of asymptotically efficient equilibria of $\Gamma^{\delta}(G)$.

Lemma 2. Suppose that ij is a $\tilde{G}$-inefficient link with $v_{i}^{*}(\tilde{G})=v_{j}^{*}(\tilde{G})=1 / 2$. Then there exists a sequence of links $l_{1} m_{1}, \ldots, l_{\bar{s}} m_{\bar{s}}$ in $\tilde{G} \ominus\{i, j\}$ with the following properties, where $\tilde{G}_{s}:=\tilde{G} \ominus\left\{l_{1}, m_{1}, \ldots, l_{s-1}, m_{s-1}\right\}$, 
(1) for $s=1, \ldots, \bar{s}, l_{s} m_{s}$ is $\tilde{G}_{s}$-efficient, and $p^{*}\left(\tilde{G}_{s} ; l_{s} m_{s}\right)=1$;

(2) $\tilde{G}_{\bar{s}+1}$ is perfect.

Lemma 3. Suppose that $\tilde{G}$ is a perfect network such that ij is $\tilde{G}$-inefficient. Then there exists a sequence of links $l_{1} m_{1}, \ldots, l_{\bar{s}} m_{\bar{s}}$ in $\tilde{G} \ominus\{i, j\}$ with the following properties, where $\tilde{G}_{s}:=\tilde{G} \ominus\left\{l_{1}, m_{1}, \ldots, l_{s-1}, m_{s-1}\right\}$,

(1) for $s=1, \ldots, \bar{s}, \tilde{G}_{s}$ is non-trivial perfect and $\left(l_{s}, m_{s}\right) \in\left\{\left(h\left(\tilde{G}_{s}\right), i\left(\tilde{G}_{s}\right)\right),\left(k\left(\tilde{G}_{s}\right), j\left(\tilde{G}_{s}\right)\right)\right\}$;

(2) $j \in\left\{h\left(\tilde{G}_{\bar{s}+1}\right), i\left(\tilde{G}_{\bar{s}+1}\right), j\left(\tilde{G}_{\bar{s}+1}\right), k\left(\tilde{G}_{\bar{s}+1}\right)\right\}$.

Remark 2. If $i j$ is a $\tilde{G}$-inefficient link, then $i j$ is also a $\tilde{\tilde{G}}$-inefficient link, and consequently $i$ and $j$ are always matched in $\tilde{\tilde{G}}$, for any subnetwork $\tilde{\tilde{G}}$ of $\tilde{G}$ obtained via successive removal of efficient links $l m$ with $\{l, m\} \cap\{i, j\}=\emptyset$, as in Lemmata 2 and 3. It follows that if $i j$ is a $\tilde{G}$-inefficient link with $v_{i}^{*}(\tilde{G})=v_{j}^{*}(\tilde{G})=1 / 2$, then there exists a sequence of links in $\tilde{G} \ominus\{i, j\}$ corresponding in order to Lemmata 2 and 3 ,

$$
l_{1} m_{1}, \ldots, l_{s_{1}} m_{s_{1}}, l_{s_{1}+1} m_{s_{1}+1}, \ldots, l_{s_{2}} m_{s_{2}}
$$

with associated subnetworks $\tilde{G}_{s}:=\tilde{G} \ominus\left\{l_{1}, m_{1}, \ldots, l_{s-1}, m_{s-1}\right\}$, satisfying the following conditions:

(1) $p^{*}\left(\tilde{G}_{s} ; l_{s} m_{s}\right)=1$ for $s=1, \ldots, s_{1}$ (corresponding to Lemma 2)

(2) $\left(l_{s}, m_{s}\right) \in\left\{\left(h\left(\tilde{G}_{s}\right), i\left(\tilde{G}_{s}\right)\right),\left(k\left(\tilde{G}_{s}\right), j\left(\tilde{G}_{s}\right)\right)\right\}$ for $s=s_{1}+1, \ldots, s_{2}$ (corresponding to

\section{Lemma 3 )}

(3) $\tilde{G}_{s_{2}+1}$ is perfect and $j \in\left\{h\left(\tilde{G}_{s_{2}+1}\right), i\left(\tilde{G}_{s_{2}+1}\right), j\left(\tilde{G}_{s_{2}+1}\right), k\left(\tilde{G}_{s_{2}+1}\right)\right\}$.

Such sequences play a critical role in the construction of reward paths in the proof of Theorem 1 below. They appear in various subnetworks $\tilde{G} \in \mathcal{G}$ and for different values of $i, j \in \tilde{G}$.

The following two lemmata are used in designing punishment paths in the construction of asymptotically efficient equilibria of $\Gamma^{\delta}(G)$.

Lemma 4. Suppose that $\tilde{G}$ is a perfect network such that ij is $\tilde{G}$-inefficient and $j k$ is $\tilde{G}$ efficient. Then there exist $h$ such that $h-i-j-k$ form a core configuration in $\tilde{G}$, and a sequence of links $l_{1} m_{1}, \ldots, l_{\bar{s}} m_{\bar{s}}$ in $\tilde{G} \ominus\{h, i, j, k\}$ with the following properties, where $\tilde{G}_{s}:=\tilde{G} \ominus\left\{l_{1}, m_{1}, \ldots, l_{s-1}, m_{s-1}\right\}$,

(1) for $s=1, \ldots, \bar{s}, l_{s} m_{s}$ is $\tilde{G}_{s}$-efficient, and $m_{s}$ has a $\tilde{G}_{s}$-efficient link to either $i$ or $j$; 
(2) the set of players in $\tilde{G}_{\bar{s}+1} \ominus\{h, i, j, k\}$ is $\tilde{G}_{\bar{s}+1}$-efficiently closed.

Lemma 5. Suppose that the set of players in $\tilde{G} \ominus\{h, i, j, k\}$ is $\tilde{G}$-efficiently closed. Then there exists a sequence of links $l_{1} m_{1}, \ldots, l_{\bar{s}} m_{\bar{s}}$ in $\tilde{G} \ominus\{h, i, j, k\}$ with the following properties, where $\tilde{G}_{s}:=\tilde{G} \ominus\left\{l_{1}, m_{1}, \ldots, l_{s-1}, m_{s-1}\right\}$,

(1) for $s=1, \ldots, \bar{s}, l_{s} m_{s}$ is $\tilde{G}_{s}$-efficient, and $p^{*}\left(\tilde{G}_{s} ; l_{s} m_{s}\right)=1$;

(2) all players different from $h, i, j, k$ are isolated in the network $\tilde{G}_{\bar{s}+1}$.

Remark 3. Suppose that $\tilde{G}$ is a perfect network such that $i j$ is $\tilde{G}$-inefficient and $j k$ is $\tilde{G}$-efficient. Let $h$ be $i$ 's match in an arbitrary efficient match of $\tilde{G}$ that includes the link $j k$. Then $\tilde{G}$ and $h, i, j, k$ satisfy the hypotheses of Lemma 4 . Hence there exists a sequence of links in $\tilde{G} \ominus\{h, i, j, k\}$ corresponding in order to Lemmata 4 and 5 ,

$$
l_{1} m_{1}, \ldots, l_{s_{1}} m_{s_{1}}, l_{s_{1}+1} m_{s_{1}+1}, \ldots, l_{s_{2}} m_{s_{2}},
$$

with associated subnetworks $\tilde{G}_{s}:=\tilde{G} \ominus\left\{l_{1}, m_{1}, \ldots, l_{s-1}, m_{s-1}\right\}$, satisfying the following conditions:

(1) $l_{s} m_{s}$ is $\tilde{G}_{s}$-efficient for $s=1, \ldots, s_{2}$

(2) $m_{s}$ has a $\tilde{G}_{s}$-efficient link to $i$ or $j$ for $s=1, \ldots, s_{1}$ (corresponding to Lemma 4)

(3) $p^{*}\left(\tilde{G}_{s} ; l_{s} m_{s}\right)=1$ for $s=s_{1}+1, \ldots, s_{2}$ (corresponding to Lemma 5)

(4) all players different from $h, i, j, k$ are isolated in $\tilde{G}_{s_{2}+1}$

Such sequences play a critical role in the construction of punishment paths in the proof of Theorem 1 below. They appear in various subnetworks $\tilde{G} \in \mathcal{G}$ and for different values of $i, j, k \in \tilde{G}$.

We are now prepared to state and prove the main result.

Theorem 1. There exists $\underline{\delta}$ so that for $\delta>\underline{\delta}$ the bargaining game $\Gamma^{\delta}(G)$ admits an equilibrium $\sigma^{* \delta}(G)$ that yields expected payoffs identical to the $G$-quasi-Markov payoffs $v^{* \delta}(G)$. The family of equilibria $\left(\sigma^{* \delta}(G)\right)_{\delta \in(\underline{\delta}, 1)}$ is asymptotically efficient.

Proof. We prove the first part of the theorem by contradiction. One immediate consequence of its negation is that there exists a well-behaved sequence of discount factors $\left(\delta_{\alpha}\right)_{\alpha \geq 0}$ converging to 1 such that $\Gamma^{\delta_{\alpha}}(G)$ does not admit an equilibrium with expected payoffs $v^{* \delta_{\alpha}}(G)$ 
for any $\alpha \geq 0$. Fix such a sequence, and denote the limits of $\left(v_{g}^{* \delta_{\alpha}}(\tilde{G})\right)_{\alpha \geq 0}$ and $\left(p^{* \delta_{\alpha}}(\tilde{G} ; i j)\right)_{\alpha \geq 0}$ as $\alpha \rightarrow \infty$ by $v_{g}^{*}(\tilde{G})$ and $p^{*}(\tilde{G} ; i j)$, respectively, for all relevant $g, i, j, \tilde{G}$. In order to reach a contradiction, the main part of the proof below details the construction of a strategy profile $\sigma^{* \delta_{\alpha}}(G)$ which for sufficiently large $\alpha$ constitutes an equilibrium of $\Gamma^{\delta_{\alpha}}(G)$ with expected payoffs $v^{* \delta_{\alpha}}(G)$.

For each $\alpha \geq 0, \sigma^{* \delta_{\alpha}}(G)$ is based on the $\operatorname{MPE} \bar{\sigma}^{* \delta_{\alpha}}(G)$ of the modified bargaining game $\bar{\Gamma}^{\delta_{\alpha}}(G)$. By definition $\bar{\sigma}^{* \delta_{\alpha}}(G)$ satisfies the incentive constraints for $\bar{\Gamma}^{\delta_{\alpha}}(G)$, and we wish to exploit this fact in our equilibrium construction for $\Gamma^{\delta_{\alpha}}(G)$. However, $\bar{\Gamma}^{\delta_{\alpha}}(G)$ differs from $\Gamma^{\delta_{\alpha}}(G)$ in subgames with induced subnetwork $\tilde{G}$ by payoff modifications that in equilibrium

(1) "prohibit" agreements across $\tilde{G}$-inefficient links;

(2) "impose" agreements when players $h(\tilde{G})$ and $k(\tilde{G})$ are selected to propose to $i(\tilde{G})$ and respectively $j(\tilde{G})$ (if $\tilde{G}$ is non-trivial perfect).

If the equilibrium $\sigma^{* \delta_{\alpha}}(G)$ is to be constructed "on top of" $\bar{\sigma}^{* \delta_{\alpha}}(G)$ we need to modify the latter in a non-Markovian fashion so as to be able to make disagreement incentive compatible in case (1) and agreement incentive compatible in case (2) without recourse to artificial payoff modifications. We achieve this by rewarding players for resisting "tempting" offers across inefficient links, and conversely by punishing the particular players who do not make the offers required to achieve agreement or deliver the promised rewards across efficient links as the case might be.

After any history that induces the network $\tilde{G}$, the constructed equlibrium may be in one of three sorts of regimes. In the default regime for $\tilde{G}$ behavior conforms to $\bar{\sigma}^{\delta_{\alpha}}(\tilde{G})$. The $i$ tempted $j$ regime for $\tilde{G}$ rewards a player $j$ in case he rejects a tempting offer (relative to his limit $\tilde{G}$-quasi-Markov payoff) from $i$ when $i j$ is a $\tilde{G}$-inefficient link. The $j$ punishes $k$ regime for $\tilde{G}$ penalizes player $k$ and benefits player $j$ (relative to their corresponding limit $\tilde{G}$-quasi-Markov payoffs) in case $k$ refuses to follow some behavior prescribed by either of the three regimes (e.g., rewarding $j$ in the $i$ tempted $j$ regime for $\tilde{G}$, or reaching an agreement with $j=j(\tilde{G})$ for $k=k(\tilde{G})$ in the default regime for $\tilde{G})$. The definitions of the latter two regimes are restricted to sets of $\tilde{G}, i, j$ and respectively $\tilde{G}, j, k$ specified later.

We argue below that the payoffs delivered by the three regimes are as follows. Set $\varepsilon=$ $\left(2 e^{G}\right)^{-e^{G}} / 200$ independently of $\alpha$. The default regime for $\tilde{G}$ yields payoffs identical to 
the $\tilde{G}$-quasi-Markov payoffs $v^{* \delta_{\alpha}}(\tilde{G})$. The $i$ tempted $j$ regime for $\tilde{G}$ yields a limit payoff above $1 / 2+\left(2 e^{G}\right)^{-e^{G}} \varepsilon$ for $j$ as $\alpha \rightarrow \infty$, and a payoff identical to the $\tilde{G}$-quasi-Markov payoff $v_{i}^{* \delta_{\alpha}}(\tilde{G})$ for $i$. The $j$ punishes $k$ regime for $\tilde{G}$ yields limit payoffs below $1 / 2-\left(2 e^{G}\right)^{-e^{G}} / 100$ for $k$ and above $1 / 2+\left(2 e^{G}\right)^{-e^{G}} / 100$ for $j$ as $\alpha \rightarrow \infty$.

In our construction, first period play is according to the default regime for $G$. The default regime is, in equilibrium-i.e., when players do not deviate-an absorbing regime. Consequently strategies in the default regime for $\tilde{G}$ determine a distribution over bargaining outcomes in $\Gamma^{\delta_{\alpha}}(\tilde{G})$ identical to the distribution induced by the $\operatorname{MPE} \bar{\sigma}^{* \delta_{\alpha}}(\tilde{G})$ in $\bar{\Gamma}^{\delta_{\alpha}}(\tilde{G})$, and yield payoffs equal to the $\tilde{G}$-quasi-Markov payoffs $v^{* \delta_{\alpha}}(\tilde{G})$. Moreover, in the default regime, deviations other than those arising in the cases (1) and (2) above are ignored. That is, they do not result in a change of regime. These facts greatly simplify the checking of incentives below.

In the default regime for $\tilde{G}$ strategies are as follows. Suppose $i$ is selected to make an offer to $j$. If $i j$ is $\tilde{G}$-efficient, then the regime specifies that $i$ and $j$ behave according to the first period strategies induced by $\bar{\sigma}^{* \delta_{\alpha}}(\tilde{G})$ in $\bar{\Gamma}^{\delta_{\alpha}}(\tilde{G})$; in case $\tilde{G}$ is non-trivial perfect and $(i, j) \in\{(h(\tilde{G}), i(\tilde{G})),(k(\tilde{G}), j(\tilde{G}))\}$ (corresponding to case (1) above), if an offer from $i$ smaller than $\delta_{\alpha} v_{j}^{* \delta_{\alpha}}(\tilde{G})$ is rejected by $j$, then play switches to the $j$ punishes $i$ regime for $\tilde{G}$. If $i j$ is $\tilde{G}$-inefficient with $v_{i}^{*}(\tilde{G})+v_{j}^{*}(\tilde{G})>1$, then the regime specifies that $i$ offer 0 and $j$ accept only offers greater than $\delta_{\alpha} v_{j}^{* \delta_{\alpha}}(\tilde{G})$. If $i j$ is $\tilde{G}$-inefficient with $v_{i}^{*}(\tilde{G})+v_{j}^{*}(\tilde{G}) \leq 1$ (effectively corresponding to the "tempting" circumstances of case (2) above), then the regime specifies that $i$ offer 0 and $j$ accept only offers greater than or equal to $1 / 2+\varepsilon^{2}$; following any offer from $i$ in the interval $\left(0,1 / 2+\varepsilon^{2}\right)$ rejected by $j$ play switches to the $i$ tempted $j$ regime for $\tilde{G}$. The two new regimes are defined below.

Players do not have incentives to make one shot deviations in $\Gamma^{\delta_{\alpha}}(\tilde{G})$ from the behavior prescribed by the default regime for $\tilde{G}$ in bargaining encounters for which no action can lead to an exit from the default regime. This is because $\bar{\sigma}^{* \delta_{\alpha}}(\tilde{G})$ is an MPE of $\bar{\Gamma}^{\delta_{\alpha}}(\tilde{G})$ with payoffs $v^{* \delta_{\alpha}}(\tilde{G})$, and compliance with the default regime for $\tilde{G}$ also yields payoffs $v^{* \delta_{\alpha}}(\tilde{G})$. For large $\alpha$, this includes the case of $\tilde{G}$-inefficient links $i j$ with $v_{i}^{*}(\tilde{G})+v_{j}^{*}(\tilde{G})>1$. For such $i$ and $j$, player $j$ 's response is optimal because rejection of any offer leads to the default regime for $\tilde{G}$, where his continuation payoff is $\delta_{\alpha} v_{j}^{* \delta_{\alpha}}(\tilde{G})$. Player $i$ has incentives to make an unacceptable offer because any agreement would obtain him less than $1-\delta_{\alpha} v_{j}^{* \delta_{\alpha}}(\tilde{G})$, while disagreement 
leads to the default regime for $\tilde{G}$, where his continuation payoff is $\delta_{\alpha} v_{i}^{* \delta_{\alpha}}(\tilde{G})$. The condition $v_{i}^{*}(\tilde{G})+v_{j}^{*}(\tilde{G})>1$ implies that $1-\delta_{\alpha} v_{j}^{* \delta_{\alpha}}(\tilde{G})<\delta_{\alpha} v_{i}^{* \delta_{\alpha}}(\tilde{G})$ for large $\alpha$.

We next address the incentive problems in $\Gamma^{\delta_{\alpha}}(\tilde{G})$ for the default regime for $\tilde{G}$ in bargaining encounters that necessitate non-default responses. Consider first the case in which $k(\tilde{G})$ is selected to make an offer to $j(\tilde{G})$ where $\tilde{G}$ is a non-trivial perfect network. ${ }^{8}$ Note that $\bar{\sigma}^{* \delta_{\alpha}}(\tilde{G})$ must specify that $k(\tilde{G})$ offer $\delta_{\alpha} v_{j(\tilde{G})}^{* \delta_{\alpha}}(\tilde{G})$ to $j(\tilde{G})$, and $j(\tilde{G})$ accept any offers at least as large with probability 1. By Proposition 3, since $\tilde{G}$ is a non-trivial perfect network, $\lim _{\alpha \rightarrow \infty} \delta_{\alpha} v_{j(\tilde{G})}^{* \delta_{\alpha}}(\tilde{G})=1 / 2$. For large $\alpha$, player $j(\tilde{G})$ has incentives to follow the behavior prescribed by the default regime for $\tilde{G}$ since rejection of offers smaller than $\delta_{\alpha} v_{j(\tilde{G})}^{* \delta_{\alpha}}(\tilde{G})$ yields the $j(\tilde{G})$ punishes $k(\tilde{G})$ regime for $\tilde{G}$ limit payoff larger than $1 / 2+\left(2 e^{G}\right)^{-e^{G}} / 100$, while rejection of offers larger than or equal to $\delta_{\alpha} v_{j(\tilde{G})}^{* \delta_{\alpha}}(\tilde{G})$ yields the default regime for $\tilde{G}$ payoff of $\delta_{\alpha} v_{j(\tilde{G})}^{* \delta_{\alpha}}(\tilde{G})$. For large $\alpha$, player $k(\tilde{G})$ has incentives to follow the behavior prescribed by the default regime for $\tilde{G}$ because offers larger than or equal to $\delta_{\alpha} v_{j(\tilde{G})}^{* \delta_{\alpha}}(\tilde{G})$ are accepted, while smaller offers are rejected leading to the $j(\tilde{G})$ punishes $k(\tilde{G})$ regime for $\tilde{G}$ limit payoff smaller than $1 / 2-\left(2 e^{G}\right)^{-e^{G}} / 100$.

Consider now the case in which $i$ is selected to make an offer to $j$, when $i j$ is $\tilde{G}$-inefficient with $v_{i}^{*}(\tilde{G})+v_{j}^{*}(\tilde{G}) \leq 1$. As $i j$ is $\tilde{G}$-inefficient, $i$ and $j$ are always efficiently matched in $\tilde{G}$, and thus $v_{i}^{*}(\tilde{G}), v_{j}^{*}(\tilde{G}) \geq 1 / 2$ by Proposition 3 . Consequently, $v_{i}^{*}(\tilde{G})=v_{j}^{*}(\tilde{G})=1 / 2$. For large $\alpha$, player $j$ has incentives to reject any offer in $\left(0,1 / 2+\varepsilon^{2}\right)$ from player $i$ since his limit payoff in the $i$ tempted $j$ regime for $\tilde{G}$ is greater than $1 / 2+\left(2 e^{G}\right)^{-e^{G}} \varepsilon$, and $1 / 2+\left(2 e^{G}\right)^{-e^{G}} \varepsilon>1 / 2+\varepsilon^{2}$ (recall that $\left.\varepsilon=\left(2 e^{G}\right)^{-e^{G}} / 200\right)$. For large $\alpha, \delta_{\alpha} v_{i}^{* \delta_{\alpha}}(\tilde{G})>1 / 2-\varepsilon^{2}$, so $i$ does not have incentives to make an acceptable offer to $j$. Also, $i$ cannot strictly benefit by making unacceptable offers to $j$ that trigger the $i$ tempted $j$ regime for $\tilde{G}$ since his expected payoff in that case is $\delta_{\alpha} v_{i}^{* \delta_{\alpha}}(\tilde{G})$.

The $i$ tempted $j$ regime for $\tilde{G}$ is defined for $i j \in \tilde{G} \in \mathcal{G}$ such that $i j$ is $\tilde{G}$-inefficient with $v_{i}^{*}(\tilde{G})=v_{j}^{*}(\tilde{G})=1 / 2$. For such $i, j, \tilde{G}$, there exists a sequence of links in $\tilde{G} \ominus\{i, j\}$ corresponding in order to Lemmata 2 and 3 ,

$$
l_{1} m_{1}, \ldots, l_{s_{1}} m_{s_{1}}, l_{s_{1}+1} m_{s_{1}+1}, \ldots, l_{s_{2}} m_{s_{2}}
$$

\footnotetext{
${ }^{8}$ The case in which $h(\tilde{G})$ is selected to make an offer to $i(\tilde{G})$ can be treated analogously.
} 
with associated subnetworks $\tilde{G}_{s}:=\tilde{G} \ominus\left\{l_{1}, m_{1}, \ldots, l_{s-1}, m_{s-1}\right\}$, satisfying the properties enumerated in Remark 2. Let $\bar{s}:=s_{2}+1$. To fix ideas, assume that $j \in\left\{j\left(\tilde{G}_{\bar{s}}\right), k\left(\tilde{G}_{\bar{s}}\right)\right\}$. Let $\{k\}=\left\{j\left(\tilde{G}_{\bar{s}}\right), k\left(\tilde{G}_{\bar{s}}\right)\right\} \backslash\{j\}$. We add the link $l_{\bar{s}} m_{\bar{s}}$ with $l_{\bar{s}}=k\left(\tilde{G}_{\bar{s}}\right), m_{\bar{s}}=j\left(\tilde{G}_{\bar{s}}\right)$ to the sequence.

Player $j$ is rewarded by player $k$ (relative to his limit $\tilde{G}$-quasi-Markov payoffs) in period $\bar{s}$ of the regime only along the path on which nature selects $l_{s}$ to make an offer to $m_{s}$ in period $s$ of the regime for each $s=1, \ldots, \bar{s}$. The reward path is described by the history $h_{\bar{s}+1}$, in which in period $s$ nature selects $l_{s}$ to make an offer to $m_{s}$, and $l_{s}$ offers $\delta_{\alpha} v_{m_{s}}^{* \delta_{\alpha}}\left(\tilde{G}_{s}\right)$ for $s<\bar{s}$, $l_{\bar{s}}$ offers $1 / 2+\varepsilon$ or $1 / 2-\varepsilon$ depending on whether $l_{\bar{s}}$ is $k$ or respectively $j$, and $m_{s}$ accepts the offer in each case. Denote by $h_{s}$ the truncation of $h_{\bar{s}+1}$ to the first $s-1$ periods. For any first instance $s$ of the regime in which the play of nature or of players $l_{s}$ and $m_{s}$ deviates from $h_{s}$ in ways different from the ones emphasized below, strategies revert to the default regime for the corresponding subgame.

In the $i$ tempted $j$ regime for $\tilde{G}$ strategies are as follows. Suppose $l_{s}$ is selected to make an offer to $m_{s}$ in period $s$ of the regime. For all $s<\bar{s}$, players $l_{s}$ and $m_{s}$ behave according to the first period strategies induced by $\bar{\sigma}^{* \delta_{\alpha}}\left(\tilde{G}_{s}\right)$ in $\bar{\Gamma}^{\delta_{\alpha}}\left(\tilde{G}_{s}\right)$. Then behavior along the reward path is identical to play in the corresponding default regime. The regime specifies that any observed deviation by $l_{s}$ and $m_{s}$ from these strategies triggers the response that the deviation would generate in the default regime for $\tilde{G}_{s}$. Thus $l_{s}$ and $m_{s}$ have incentives to follow the prescribed behavior in period $s$ of the $i$ tempted $j$ regime for $\tilde{G}$ because they have in the default regime for $\tilde{G}_{s}$.

For the link $l_{\bar{s}} m_{\bar{s}}$, recall that $\left\{l_{\bar{s}}, m_{\bar{s}}\right\}=\left\{j\left(\tilde{G}_{\bar{s}}\right), k\left(\tilde{G}_{\bar{s}}\right)\right\}=\{j, k\}$. We consider two cases, $l_{\bar{s}}=j$ and $l_{\bar{s}}=k$. If $l_{\bar{s}}=j$ then the regime specifies that $l_{\bar{s}}=j$ offer $1 / 2-\varepsilon$ and $m_{\bar{s}}=k$ accept any offer at least as large. If $l_{\bar{s}}=k$ then the regime specifies that $l_{\bar{s}}=k$ offer $1 / 2+\varepsilon$ and $m_{\bar{s}}=j$ accept any offer at least as large. If in the former case $k$ rejects an offer from $j$ larger than or equal to $1 / 2-\varepsilon$ or in the latter case $k$ makes an offer smaller than $1 / 2+\varepsilon$ that is rejected by $j$, then $k$ is punished relative to his limit $\tilde{G}_{\bar{s}}$-quasi-Markov payoffs by switching to the $j$ punishes $k$ regime for $\tilde{G}_{\bar{s}}$ (the regime is well-defined because $i j$ is $\tilde{G}_{\bar{s}}$-inefficient and $j k$ is $\tilde{G}_{\bar{s}}$-efficient as it is identical to $\left.j\left(\tilde{G}_{\bar{s}}\right) k\left(\tilde{G}_{\bar{s}}\right)\right)$. For large $\alpha$, players $j$ and $k$ have incentives to follow the prescribed behavior in period $\bar{s}$ of the $i$ tempted $j$ regime for $\tilde{G}$ in either case because the $j$ punishes $k$ regime for $\tilde{G}_{\bar{s}}$ yields limit payoffs below $1 / 2-\left(2 e^{G}\right)^{-e^{G}} / 100$ for 
$k$ and above $1 / 2+\left(2 e^{G}\right)^{-e^{G}} / 100$ for $j$, which satisfy $1 / 2 \pm\left(2 e^{G}\right)^{-e^{G}} / 100 \gtrless 1 / 2 \pm \varepsilon$ (recall that $\left.\varepsilon=\left(2 e^{G}\right)^{-e^{G}} / 200<\left(2 e^{G}\right)^{-e^{G}} / 100\right)$, and any deviation not considered here leads to the default regime limit payoffs of $1 / 2$ for both $k$ and $j$ (by Proposition 3 , as $\tilde{G}_{\bar{s}}$ is perfect).

The reward path and the strategies for the $i$ tempted $j$ regime for $\tilde{G}$ are constructed so that under this regime the distribution over pairs reaching agreement for any subgame is identical to the one in the corresponding default regime, ${ }^{9}$ and the only agreement reached at different terms in the two regimes involves $j$ and $k$ on the reward path. Hence, as desired, all players different from $j$ and $k$, in particular $i$, receive payoffs equal to their corresponding $\tilde{G}_{-}$ quasi-Markov payoffs. As $\alpha \rightarrow \infty$, the $i$ tempted $j$ regime for $\tilde{G}$ yields a limit payoff above $1 / 2+\left(2 e^{G}\right)^{-e^{G}} \varepsilon$ for $j$ for the following reasons. The limit payoff of $j$ is $1 / 2+\varepsilon$ along the reward path of the regime, which realizes with limit probability greater than $\left(2 e^{\tilde{G}}\right)^{-e^{\tilde{G}}} \geq\left(2 e^{G}\right)^{-e^{G}}$ $\left(e^{\tilde{G}} \leq e^{G}, \forall \tilde{G} \in \mathcal{G}\right)$, and identical to the corresponding limit quasi-Markov payoffs of at least $1 / 2$ along any other path. The latter fact is true since $j$ is always efficiently matched in $\tilde{G}$, and also in the subnetwork induced by any subgame off the reward path, where players behave according to the corresponding default regime. Thus $j$ receives corresponding limit quasi-Markov payoffs of at least $1 / 2$ in such subgames, by the first part of Proposition 3.

The $j$ punishes $k$ regime for $\tilde{G}$ is defined for perfect networks $\tilde{G} \in \mathcal{G}$ for which $j k$ is $\tilde{G}$-efficient and there exists $i$ such that $i j$ is $\tilde{G}$-inefficient. Consequently, there exists $h$ such that $h-i-j-k$ form a core configuration in $\tilde{G}$. For such $h, i, j, k, \tilde{G}$, there exist a sequence of links in $\tilde{G} \ominus\{h, i, j, k\}$ corresponding in order to Lemmata 4 and 5 ,

$$
l_{1} m_{1}, \ldots, l_{s_{1}} m_{s_{1}}, l_{s_{1}+1} m_{s_{1}+1}, \ldots, l_{s_{2}} m_{s_{2}}
$$

with associated subnetworks $\tilde{G}_{s}:=\tilde{G} \ominus\left\{l_{1}, m_{1}, \ldots, l_{s-1}, m_{s-1}\right\}$, satisfying the properties enumerated in Remark 3. Let $\bar{s}:=s_{2}+1$. We add the link $l_{\bar{s}} m_{\bar{s}}$ with $l_{\bar{s}}=j, m_{\bar{s}}=k$ to the sequence.

Player $k$ is punished by player $j$ (relative to his limit $\tilde{G}$-quasi-Markov payoff) in period $\bar{s}$ of the regime only along the path on which nature selects $l_{s}$ to make an offer to $m_{s}$ in period $s$ of the regime for each $s=1, \ldots, \bar{s}$. The reward path is described by the history $h_{\bar{s}+1}$, in which in period $s$ nature selects $l_{s}$ to make an offer to $m_{s}$, and for $s<\bar{s}, l_{s}$ offers $\min \left(1-\delta_{\alpha} v_{l_{s}}^{* \delta_{\alpha}}\left(\tilde{G}_{s}\right), \delta_{\alpha} v_{m_{s}}^{* \delta_{\alpha}}\left(\tilde{G}_{s}\right)\right)$ or $\delta_{\alpha} v_{m_{s}}^{* \delta_{\alpha}}\left(\tilde{G}_{s}\right)$ depending on whether $l_{s} m_{s}$ is a Lemma 4 or

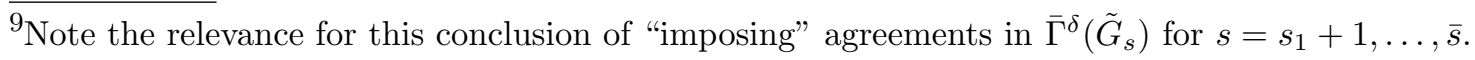


respectively Lemma 5 link, $l_{\bar{s}}=j$ offers $1 / 2-1 / 100$, and $m_{s}$ accepts the offer in each case. Denote by $h_{s}$ the truncation of $h_{\bar{s}+1}$ to the first $s-1$ periods. For any first instance $s$ of the regime in which the play of nature or of players $l_{s}$ and $m_{s}$ deviates from $h_{s}$ in ways different from the ones emphasized below, strategies revert to the default regime for the corresponding subgame.

In the $j$ punishes $k$ regime for $\tilde{G}$ strategies are as follows. Suppose $l_{s}$ is selected to make an offer to $m_{s}$ in period $s$ of the regime. For Lemma $4 \operatorname{links} l_{s} m_{s}$, the regime specifies that $l_{s}$ offer $\min \left(1-\delta_{\alpha} v_{l_{s}}^{* \delta_{\alpha}}\left(\tilde{G}_{s}\right), \delta_{\alpha} v_{m_{s}}^{* \delta_{\alpha}}\left(\tilde{G}_{s}\right)\right)$ and $m_{s}$ accept any offer at least as large. By the definition of Lemma 4 links, $m_{s}$ has a $\tilde{G}_{s}$-efficient link to either $i$ or $j$. Suppose that $m_{s}$ is $\tilde{G}_{s}$-efficiently linked to $j$ (a similar construction of the strategies is needed for $j$ replaced by $i$ ). To account for $m_{s}$ 's non-default response behavior, the punsihemet regime specifies that if an offer from $l_{s}$ greater than or equal to $\min \left(1-\delta_{\alpha} v_{l_{s}}^{* \delta_{\alpha}}\left(\tilde{G}_{s}\right), \delta_{\alpha} v_{m_{s}}^{* \delta_{\alpha}}\left(\tilde{G}_{s}\right)\right)$ is rejected by $m_{s}$, then $m_{s}$ is punished relative to his limit $\tilde{G}_{s}$-quasi-Markov payoffs by switching to the $j$ punishes $m_{s}$ regime for $\tilde{G}_{s}$ (the regime is well-defined because $i j$ is $\tilde{G}_{s}$-inefficient and $j m_{s}$ is $\tilde{G}_{s}$-efficient). The optimality of $l_{s}$ 's offer of $\min \left(1-\delta_{\alpha} v_{l_{s}}^{* \delta_{\alpha}}\left(\tilde{G}_{s}\right), \delta_{\alpha} v_{m_{s}}^{* \delta_{\alpha}}\left(\tilde{G}_{s}\right)\right)$ to $m_{s}$ given $m_{s}$ 's response strategy, and of $m_{s}$ 's rejection of smaller offers are immediately checked. ${ }^{10}$ By construction, $\tilde{G}_{s}$ is a perfect network, which coupled with the second part of Proposition 3 implies that $\lim _{\alpha \rightarrow \infty} \min \left(1-\delta_{\alpha} v_{l_{s}}^{* \delta_{\alpha}}\left(\tilde{G}_{s}\right), \delta_{\alpha} v_{m_{s}}^{* \delta_{\alpha}}\left(\tilde{G}_{s}\right)\right)=1 / 2$. For large $\alpha$, player $m_{s}$ has incentives to accept offers from $l_{s}$ greater than or equal to $\min \left(1-\delta_{\alpha} v_{l_{s}}^{* \delta_{\alpha}}\left(\tilde{G}_{s}\right), \delta_{\alpha} v_{m_{s}}^{* \delta_{\alpha}}\left(\tilde{G}_{s}\right)\right)$ since his limit payoff in the $j$ punishes $m_{s}$ regime for $\tilde{G}_{s}$ is smaller than $1 / 2-\left(2 e^{G}\right)^{-e^{G}} / 100$ as $\alpha \rightarrow \infty$.

For Lemma 5 links $l_{s} m_{s}$, the punishment regime specifies that players $l_{s}$ and $m_{s}$ behave according to the first period strategies induced by $\bar{\sigma}^{* \delta_{\alpha}}\left(\tilde{G}_{s}\right)$ in $\bar{\Gamma}^{\delta_{\alpha}}\left(\tilde{G}_{s}\right)$. Incentives for players $l_{s}$ and $m_{s}$ to follow the prescribed behavior are provided as for the case of Lemmata 2 and 3 links in the $i$ tempted $j$ regime for $\tilde{G}$.

Recall that all players different from $h, i, j, k$ are isolated in $\tilde{G}_{\bar{s}}$, and that $i j$ is $\tilde{G}_{\bar{s}}$-inefficient. Consequently, $h$ and $k$ are not connected in $\tilde{G}_{\bar{s}}$. There are 4 possible link configurations that players $h, i, j, k$ induce in $\tilde{G}_{\bar{s}}$, depending on which subset of the links $i k$ and $j h$ is included in $\tilde{G}_{\bar{s}}$. One key observation used below is that in each of the four cases, for every $\delta \in(0,1)$, the

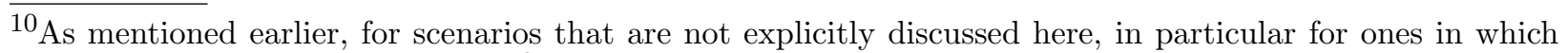
$l_{s}$ offers $m_{s}$ less than $\min \left(1-\delta_{\alpha} v_{l_{s}}^{* \delta_{\alpha}}\left(\tilde{G}_{s}\right), \delta_{\alpha} v_{m_{s}}^{* \delta_{\alpha}}\left(\tilde{G}_{s}\right)\right)$, play reverts to the default regime for the resulting subgame.
} 
game $\bar{\Gamma}^{\delta}\left(\tilde{G}_{\bar{s}}\right)$ has a unique MPE, yielding identical $\tilde{G}_{\bar{s}^{-}}$quasi-Markov payoffs, strictly smaller than $1 / 2$, for all four players. We conclude that $\delta_{\alpha}\left(v_{i}^{* \delta_{\alpha}}\left(\tilde{G}_{\bar{s}}\right)+v_{j}^{* \delta_{\alpha}}\left(\tilde{G}_{\bar{s}}\right)\right)<1$ for every $\alpha \geq 0$.

For the link $l_{\bar{s}} m_{\bar{s}}$, with $l_{\bar{s}}=j$ and $m_{\bar{s}}=k$, the punishment regime specifies that $j$ offer $1 / 2-1 / 100$ and $k$ accept any offer at least as large. Suppose that $k$ rejects an offer greater than or equal to $1 / 2-1 / 100$. For this deviation, the equilibrium specifies that if nature selects $i$ to make an offer to $j$ next period, then $i$ offers $\delta_{\alpha} v_{j}^{* \delta_{\alpha}}\left(\tilde{G}_{\bar{s}}\right)$ and $j$ accepts any offer at least as large. Players $i$ and $j$ have incentives to implement the isolation of $h$ and $k$ in $\tilde{G}_{\bar{s}}$ because $\delta_{\alpha}\left(v_{i}^{* \delta_{\alpha}}\left(\tilde{G}_{\bar{s}}\right)+v_{j}^{* \delta_{\alpha}}\left(\tilde{G}_{\bar{s}}\right)\right)<1$. If nature does not select $i$ to make an offer to $j$, or $i$ and $j$ deviate from the described strategies then play reverts to the default regime for the subsequent subgame. Thus $k$ is punished by isolation in the event that $i$ is selected to make an offer to $j\left(h k \notin \tilde{G}_{\bar{s}}\right)$, which occurs with probability of at least $1 / 10$, as $\tilde{G}_{\bar{s}}$ has at most 5 links. As $\alpha \rightarrow \infty$, the limit payoff of $k$ is 0 along the one-period isolation path, and $1 / 2$ along any other path, by the second part of Proposition 3. For large $\alpha$, player $k$ has incentives to accept offers greater than or equal to $1 / 2-1 / 100$ from $j$ since his limit payoff conditional on rejecting such offers is at most $1 / 2(1-1 / 10)$. The optimality of $j$ 's offer of $1 / 2-1 / 100$ to $k$ given $k$ 's response strategy, and of $k$ 's rejection of smaller offers are immediately checked (similarly to footnote 10).

As $\alpha \rightarrow \infty$, the $j$ punishes $k$ regime for $\tilde{G}$ yields limit payoffs below $1 / 2-\left(2 e^{G}\right)^{-e^{G}} / 100$ for $k$ and above $1 / 2+\left(2 e^{G}\right)^{-e^{G}} / 100$ for $j$ for the following reasons. The limit payoffs of $j$ and $k$ are $1 / 2+1 / 100$ and respectively $1 / 2-1 / 100$ along the punishment path, which realizes with limit probability greater than $\left(2 e^{\tilde{G}}\right)^{-e^{\tilde{G}}} \geq\left(2 e^{G}\right)^{-e^{G}}\left(e^{\tilde{G}} \leq e^{G}, \forall \tilde{G} \in \mathcal{G}\right)$, and identical to the corresponding limit quasi-Markov payoffs of $1 / 2$ along any other path. The latter fact is true since $\tilde{G}$ is perfect, and so is any subnetwork induced by any subgame off the punishment path, where players behave according to the corresponding default regime. Thus $j$ and $k$ receive corresponding limit quasi-Markov payoffs of $1 / 2$ in such subgames, by the second part of Proposition 3.

The constructed strategies yield payoffs $v^{* \delta_{\alpha}}(G)$ and satisfy all the equilibrium requirements in $\Gamma^{\delta_{\alpha}}(G)$ for sufficiently large $\alpha$. This contradiction with our initial assumption completes the proof of the first part of the theorem. For the second part, note that the family of equilibria whose existence is established in the first part is asymptotically efficient by Remark 1 . 
Remark 4. We can make $i$ 's incentives not to tempt $j$ strict in the equilibrium construction of Theorem 1, by delaying $j$ 's reward from $k$-and $i$ 's subsequent agreements-by one period. Specifically, $j$ receives $1 / 2+\varepsilon$ from $k$ only if matched to bargain with $k$ for two consecutive periods $(\bar{s}$ and $\bar{s}+1)$ of the $i$ tempted $j$ regime for $\tilde{G}$, following the prescribed series of agreements across Lemmata 2 and 3 links. In that case, in the second period in which the two players are matched to bargain (period $\bar{s}+1$ of the regime), $j$ receives $1 / 2+\varepsilon$ from $k$. Depending on whether $l_{\bar{s}}$ is $j$ or $k$, player $l_{\bar{s}}$ can be incentivized to make the unacceptable offer of 0 to $m_{\bar{s}}$ in the first period in which the two players are matched to bargain (period $\bar{s}$ of the regime) via the following threats. In case $l_{\bar{s}}=j$, if $j$ makes a non-zero offer to $k$ that is rejected by $k$ then play reverts to the corresponding default regime. In case $l_{\bar{s}}=k$, if $k$ makes a non-zero offer to $j$ that is rejected by $j$ then play switches to the $j$ punishes $k$ regime for the corresponding subgame.

\section{Properties of Markov Perfect Equilibria}

While MPEs may be incompatible with efficiency (as demonstrated in the preceding section), they are of independent interest. ${ }^{11}$ In this section we investigate the distinctive structure and properties of MPEs in the context of our model of bargaining in networks. We first provide examples of MPEs for some simple networks. We mainly focus on equilibrium payoffs. Strategies may be constructed as in the proof of Proposition 1.

Consider first the star networks, in which one player controls the bargaining opportunities of all other players. Formally, in the star of $n$ network $G_{\text {star } n}$ player 1 is connected to player $k$ for $k=2, \ldots, n$. Proposition 4(i) in Rubinstein and Wolinsky (1990) shows that the bargaining game $\Gamma^{\delta}\left(G_{\text {star } n}\right)$ has a unique subgame perfect equilibrium (which is also the unique MPE), with payoffs given by

$$
v_{1}^{* \delta}\left(G_{\text {star } n}\right)=\frac{n-1-\delta}{n(2-\delta)-2} \text { and } v_{k}^{* \delta}\left(G_{\text {star } n}\right)=\frac{1-\delta}{n(2-\delta)-2} \text { for } k=2, \ldots, n .
$$

As $\delta \rightarrow 1$, the equilibrium payoffs converge to $1 / 2$ for both players if $n=2$, and 1 for player 1 and 0 for all other players if $n \geq 3$.

Consider next the line networks, in which players are located on a line and can only bargain with their closest neighbors. Formally, in the line of $n$ network $G_{\text {line } n}$ player $k$ is connected

\footnotetext{
${ }^{11}$ See for example Fudenberg and Tirole (1991), and Maskin and Tirole (2001).
} 
to player $k+1$ for $k=1, \ldots, n-1$. Computing MPEs of the bargaining game for line networks is feasible for two main reasons. First, all the connected components induced by the players remaining in any subgame are line networks. Second, the number of conjectures about what first period agreements are possible in equilibrium is small because each player has at most 2 neighbors. The networks $G_{\text {line } 2}$ and $G_{\text {line } 3}$ are isomorphic ${ }^{12}$ to $G_{\text {star } 2}$ and $G_{\text {star } 3}$ respectively.

Consider now the bargaining game on the line of 4 network, $\Gamma^{\delta}\left(G_{\text {line } 4}\right)$. If players 2 and 3 reach the first agreement, players 1 and 4 are left disconnected in the ensuing subgame, and receive zero payoffs. If players 1 and 2 (3 and 4) reach the first agreement, players 3 and 4 (1 and 2) induce a subnetwork isomorphic to $G_{\text {line } 2}$ in the ensuing subgame, and receive expected payoffs of $1 / 2$ next period. For low $\delta$ there is a unique MPE of $\Gamma^{\delta}\left(G_{\text {line } 4}\right)$. In any subgame, every match ends in agreement. The unique MPE payoffs for $\delta \leq \underline{\delta} \approx .945$ are given by ${ }^{13}$

$$
v_{1}^{* \delta}\left(G_{\text {line } 4}\right)=v_{4}^{* \delta}\left(G_{\text {line } 4}\right)=\frac{6+3 \delta-2 \delta^{2}}{12(3-\delta)}, v_{2}^{* \delta}\left(G_{\text {line } 4}\right)=v_{3}^{* \delta}\left(G_{\text {line } 4}\right)=\frac{12+3 \delta-2 \delta^{2}}{12(3-\delta)} .
$$

For $\delta>\underline{\delta}$, there exists an MPE of $\Gamma^{\delta}\left(G_{\text {line } 4}\right)$ in which players 2 and 3 reach agreement with some probability $p^{* \delta} \in(0,1)$ conditional on their link being selected for bargaining. The unique MPE payoffs and $p^{* \delta}$ are given by ${ }^{14}$

$$
\begin{array}{r}
v_{1}^{* \delta}\left(G_{\text {line } 4}\right)=v_{4}^{* \delta}\left(G_{\text {line } 4}\right)=\frac{-6+5 \delta+2 \delta^{2}}{2 \delta^{2}}, v_{2}^{* \delta}\left(G_{\text {line } 4}\right)=v_{3}^{* \delta}\left(G_{\text {line } 4}\right)=\frac{1}{2 \delta} \\
p^{* \delta}=\frac{2\left(9-12 \delta+\delta^{2}+2 \delta^{3}\right)}{\delta\left(-6+5 \delta+2 \delta^{2}\right)} .
\end{array}
$$

Note that, as players become patient, the conditional probability of agreement between players 2 and 3 converges to 0 , and the MPE payoffs converge to $1 / 2$ for each player: $\lim _{\delta \rightarrow 1} p^{* \delta}=0, \lim _{\delta \rightarrow 1} v_{k}^{* \delta}\left(G_{\text {line } 4}\right)=1 / 2$ for $k=1,2,3,4$. The intuition is that players 2 and 3 could obtain payoffs greater than $1 / 2$ in the limit only by extorting players 1 and 4 via the threat of an agreement across the link $(2,3)$, which would leave 1 and 4 disconnected.

\footnotetext{
${ }^{12}$ Two networks $H=(V, E)$ and $H^{\prime}=\left(V^{\prime}, E^{\prime}\right)$ are isomorphic if there exists a bijection $g: V \rightarrow V^{\prime}$ such that $i j \in E \Longleftrightarrow g(i) g(j) \in E^{\prime}$.

${ }^{13}$ The definition of $\underline{\delta}$ and the system of equations solved by $v^{* \delta}\left(G_{\text {line }} 4\right)$ for $\delta \leq \underline{\delta}$ appear in Appendix C.

${ }^{14}$ The system of equations solved by $v^{* \delta}\left(G_{\text {line } 4}\right)$ and $p^{* \delta}$ for $\delta>\underline{\delta}$ is written out in Appendix C.
} 
Yet, players 2 and 3 cannot reach an agreement if their limit equilibrium payoffs are larger than $1 / 2 .^{15}$

For the line of $5,7,8,9, \ldots$ networks, and other more complex networks, computing MPE payoffs for the bargaining game for every $\delta$ may be a difficult task. For these networks, we focus on limit MPE payoffs and agreement behavior as players become patient.

4.1. Limit Properties of MPE. Fix a network $G$. A payoff vector $v^{*}$ is a limit equilibrium payoff (of $\Gamma^{\delta}(G)$ as $\delta \rightarrow 1$ ) if there exists a family of subgame perfect equilibria of the games $\left(\Gamma^{\delta}(G)\right)_{\delta \in(0,1)}$ with respective payoff vectors $\left(v^{* \delta}\right)_{\delta \in(0,1)}$ such that $v^{*}=\lim _{\delta \rightarrow 1} v^{* \delta}$. A limit MPE payoff is a limit equilibrium payoff corresponding to a family of MPEs. The agreement behavior induced by a Markov strategy $\sigma$ is the profile $(p(\tilde{G} ; i j))_{i j \in \tilde{G} \in \mathcal{G}}$ where $p(\tilde{G} ; i j)$ is the probability that $i$ and $j$ reach agreement under $\sigma$ conditional on being matched to bargain in a subgame that induces the subnetwork $\tilde{G}$. ${ }^{16}$ We refer to the profile $(p(G ; i j))_{i j \in G}$ corresponding to first period agreements as initial agreement behavior. An agreement profile $(p(\tilde{G} ; i j))_{i j \in \tilde{G} \in \mathcal{G}}$ is a limit MPE agreement (for $\Gamma^{\delta}(G)$ as $\left.\delta \rightarrow 1\right)$ if there exists a family of MPEs of the games $\left(\Gamma^{\delta}(G)\right)_{\delta \in(0,1)}$ with respective induced agreement behavior $\left(p^{* \delta}(\tilde{G} ; i j)\right)_{i j \in \tilde{G} \in \mathcal{G}}$ such that $p(\tilde{G} ; i j)=\lim _{\delta \rightarrow 1} p^{* \delta}(\tilde{G} ; i j)$ for all $i j \in \tilde{G} \in \mathcal{G}$.

The bargaining game for various network structures permits a bootstrap approach to computing directly limit MPE payoffs and agreement behavior as players become patient, and constructing MPEs of $\Gamma^{\delta}(G)$ for high $\delta$ that yield the determined limit payoffs and agreements as $\delta \rightarrow 1$. We use known MPE behavior in subgames (equivalent to) $\Gamma^{\delta}(\tilde{G})$ for $\tilde{G} \in \mathcal{G}^{0}$ in order to characterize equilibrium behavior in $\Gamma^{\delta}(G)$. Suppose that for every $\delta \in(0,1)$ we constructed a subgame consistent family of MPEs $\left(\sigma^{* \delta}(G \ominus\{i, j\})\right)_{i j \in G}$ for the bargaining games $\left(\Gamma^{\delta}(G \ominus\{i, j\})\right)_{i j \in G}$ with respective payoffs $\left(v^{* \delta}(G \ominus\{i, j\})\right)_{i j \in G}$.

\footnotetext{
${ }^{15}$ Similarly, all MPEs of the bargaining game on the line of 6 network, $\Gamma^{\delta}\left(G_{\text {line }} 6\right)$, are payoff equivalent. As $\delta \rightarrow 1$, the common probability of first period agreement across the links $(2,3)$ and $(4,5)$ vanishes, while agreement obtains with probability 1 across all other links. All players receive expected payoffs of $1 / 2$ in the limit. However, the properties that limit MPE payoffs are $1 / 2$ for all players, and limit probabilities of first period agreement across links $(k, k+1)$ are 0 and 1 for $k$ even and odd, respectively, do not generalize to the bargaining games on lines with an even number larger than 6 of players.

${ }^{16}$ Mathematically, $p(\tilde{G} ; i j)$ is the average of the probabilities that the random variables $\sigma_{j}\left(h_{t} ; i \rightarrow\right.$ $\left.j ; \sigma_{i}\left(h_{t} ; i \rightarrow j\right)\right)$ and $\sigma_{i}\left(h_{t} ; j \rightarrow i ; \sigma_{j}\left(h_{t} ; j \rightarrow i\right)\right)$ equal "Yes" for a history $h_{t}$ with $G\left(h_{t}\right)=\tilde{G}$. By the definition of an MPE, the distributions of the two random variables do not depend on the choice of $h_{t}$ satisfying $G\left(h_{t}\right)=\tilde{G}$.
} 
Fix a discount factor $\delta$ and a subnetwork $H$ of $G$. We set out to construct an MPE $\sigma^{* \delta}(G)$ of $\Gamma^{\delta}(G)$ that yields payoffs $v^{* \delta}(G \ominus\{i, j\})$ in subgames that induce the subnetwork $G \ominus\{i, j\}$, and generates initial agreements with probability 1 across links in $H$ and with probability 0 across other links. That is, the initial agreement behavior for $\sigma^{* \delta}(G)$ is $p^{H}$ with $p^{H}(G ; i j)=1$ for $i j \in H$, and $p^{H}(G ; i j)=0$ otherwise. By the proof of Proposition 1 , the payoff vector for $\sigma^{* \delta}(G)$ is a solution $v$ to the $n \times n$ linear system, ${ }^{17}$

$$
v_{k}=\frac{2\left(e^{G}-e^{H}\right)+e_{k}^{H}}{2 e^{G}} \delta v_{k}+\frac{1}{2 e^{G}} \sum_{\{i \mid k i \in H\}}\left(1-\delta v_{i}\right)+\frac{1}{e^{G}} \sum_{\{i j \mid i j \in H \ominus\{k\}\}} \delta v_{k}^{* \delta}(G \ominus\{i, j\}), k=\overline{1, n} .
$$

Assume that $v^{* \delta}(G \ominus\{i, j\})$ converges to $v^{*}(G \ominus\{i, j\})$ as $\delta$ goes to 1 for all $i j \in H$. Consider the $n \times n$ linear system obtained by taking the limit $\delta \rightarrow 1$ in 4.1 ,

$$
v_{k}=\frac{2\left(e^{G}-e^{H}\right)+e_{k}^{H}}{2 e^{G}} v_{k}+\frac{1}{2 e^{G}} \sum_{\{i \mid k i \in H\}}\left(1-v_{i}\right)+\frac{1}{e^{G}} \sum_{\{i j \mid i j \in H \ominus\{k\}\}} v_{k}^{*}(G \ominus\{i, j\}), k=\overline{1, n}
$$

We show that solutions of 4.1 corresponding to MPE payoffs of $\Gamma^{\delta}(G)$ for high $\delta$ lead to a solution of 4.2 , and that under some auxiliary conditions the correspondence can be reversed.

Proposition 4. Suppose that $e^{G}>1$ and let $H$ be a subnetwork of $G$. Then the systems 4.1 and 4.2 have unique solutions, denoted $v^{\delta, H}$ and $v^{H}$, respectively. The solutions $v^{\delta, H}$ and $v^{H}$ belong to $[0,1]^{n}$, and satisfy $\lim _{\delta \rightarrow 1} v^{\delta, H}=v^{H}$. If $v^{H}$ satisfies $v_{i}^{H}+v_{j}^{H}<1$ for $i j \in H$ and $v_{i}^{H}+v_{j}^{H}>1$ for $i j \in G, i j \notin H$ then there exists $\underline{\delta}<1$ such that for every $\delta \in(\underline{\delta}, 1)$ there is a Markov perfect equilibrium of $\Gamma^{\delta}(G)$ with payoffs $v^{\delta, H}$ and initial agreement behavior $p^{H}$.

The proof appears in Appendix C. An illustration of Proposition 4 is provided in the next subsection. Proposition 4 has been used in order to determine limit MPE payoffs and initial agreements for the bargaining games on the line of $5,7,8, \ldots, 12$ networks depicted in Figure 5 in Appendix C. In all diagrams, for every network, limit MPE payoffs for each player are represented next to the corresponding node, and each link is drawn as a thin, dashed, or thick line segment depending on whether the probability of initial agreement across that link in MPEs for high $\delta$ is 0 , a number in $(0,1)$ (then the limit probability as $\delta \rightarrow 1$ is mentioned next to the link), ${ }^{18}$ or 1 , respectively.

\footnotetext{
${ }^{17}$ Recall that $e^{G}$ and $e_{k}^{G}\left(e^{H}\right.$ and $\left.e_{k}^{H}\right)$ denote the total number of links and respectively the number of links of player $k$ in the network $G(H)$.

${ }^{18}$ For some links the initial agreement probabilities for $\delta<1$ may be positive, and converge to 0 as $\delta \rightarrow 1$, as in the case of the link $(2,3)$ in $G_{\text {line } 4}$.
} 

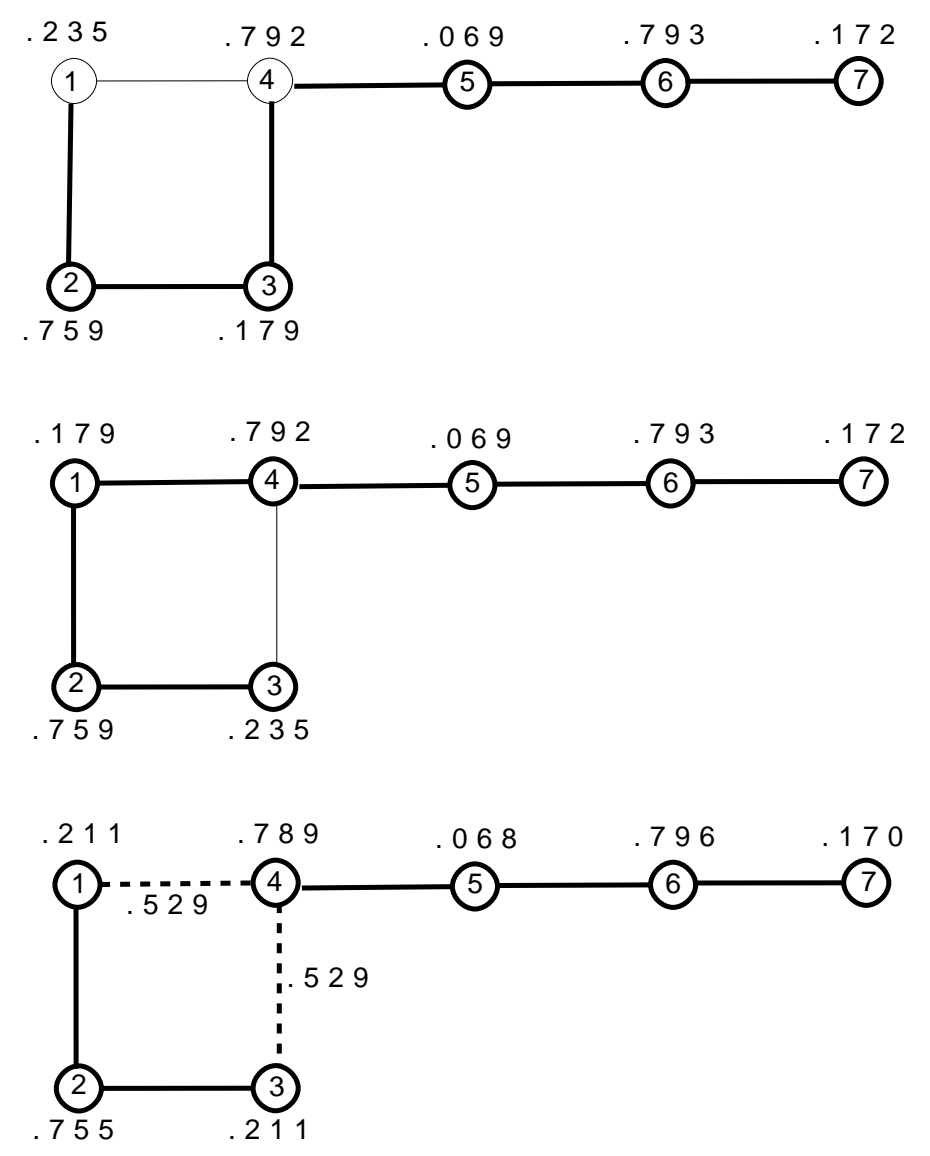

FigurE 3. Three sets of limit MPE payoffs and initial agreements for $\Gamma^{\delta}\left(G_{\text {sq+line } 3}\right)$

4.2. Multiple MPE Payoffs. Multiple MPE payoffs may exist for the bargaining game on some networks for high discount factors. One example is the bargaining game $\Gamma^{\delta}\left(G_{\text {sq+line } 3}\right)$, on the network $G_{\text {sq+line } 3}$ depicted in Figure 3.

Proposition 5. There exists $\underline{\delta}<1$ such that for every $\delta \in(\underline{\delta}, 1)$ the game $\Gamma^{\delta}\left(G_{\text {sq+line } 3}\right)$ has (at least) three MPEs that are pairwise payoff unequivalent.

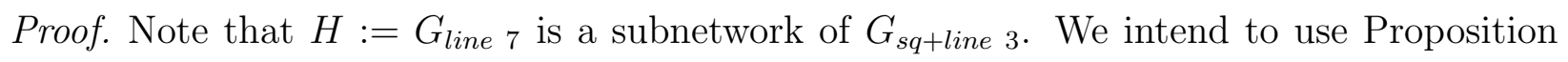
4 to show that for high $\delta$ the game $\Gamma^{\delta}\left(G_{s q+l i n e}\right)$ admits an MPE with initial agreement behavior $p^{H}$.

To define the subgame consistent collection of MPEs $\left(\sigma^{* \delta}\left(G_{s q+\text { line }} 3 \ominus\{k, k+1\}\right)\right)_{k=1,2, \ldots, 6}$ necessary for 4.1 and 4.2, note that an initial agreement between two players linked in $H$ may induce the following subgames. If players 1 and 2 (2 and 3) reach the first agreement, then players $3,4,5,6,7(1,4,5,6,7)$ induce a subgame on a network isomorphic to the line of 


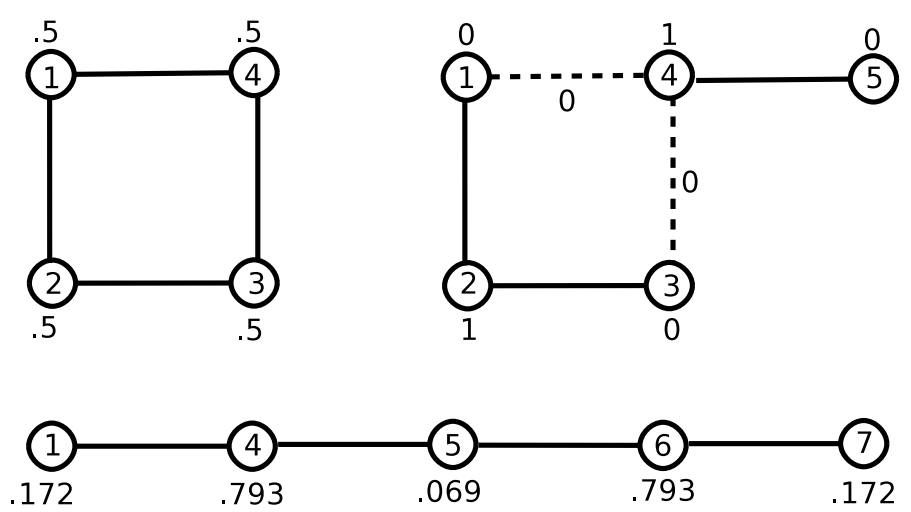

FiguRE 4. Limit MPE payoffs and initial agreements for the bargaining game on $G_{s q}$ (top left), $G_{s q+p o i n t}$ (top right), and a network isomorphic to $G_{\text {line } 5}$

5 network. If players 3 and 4 ( 4 and 5) reach the first agreement, the induced subnetwork has two connected components, partitioning the set of remaining players in $\{1,2\}$ and $\{5,6,7\}$ $(\{1,2,3\}$ and $\{6,7\})$. Players 1 and $2(6$ and 7$)$ are involved in a subgame similar to the bargaining game on the line of 2 network, with lower matching frequency since they are not matched to bargain when the links $(5,6)$ or $(6,7)((1,2)$ or $(2,3))$ are selected for bargaining. Similarly players $5,6,7(1,2,3)$ are involved in a lower matching frequency version of the bargaining game on the line of 3 network. For both lower matching frequency versions of the bargaining games on the line of 2 and 3 networks the limit MPE payoffs are identical to the ones in the respective benchmark versions. If players 5 and 6 reach the first agreement, then players 1,2,3,4 induce a subgame equivalent to the bargaining game on the square network, $G_{s q}$, and player 7 is left disconnected, in an empty game. If players 6 and 7 reach the first agreement, then players $1,2,3,4,5$ induce a subgame equivalent to the bargaining game on the square plus point network, $G_{s q+p o i n t}$. The limit MPE payoffs and initial agreements for $G_{s q}, G_{s q+p o i n t}$, and a network isomorphic to the line of 5 are summarized in Figure 4.

The limit linear system 4.2 corresponding to $\Gamma^{\delta}\left(G_{\text {sq+line } 3}\right)$ and the subnetwork $H$ is written out in Appendix C. The unique solution is $v^{H}$ with $v_{1}^{H} \approx 0.235, v_{2}^{H} \approx 0.759, v_{3}^{H} \approx$ $0.179, v_{4}^{H} \approx 0.792, v_{5}^{H} \approx 0.069, v_{6}^{H} \approx 0.793, v_{7}^{H} \approx 0.172$. The vector $v^{H}$ satisfies all the conditions from Proposition 4 , so for high $\delta$ there exists an MPE of $\Gamma^{\delta}\left(G_{\text {sq+line } 3}\right)$ with initial agreement behavior $p^{H}$ and payoffs approaching $v^{H}$ as $\delta \rightarrow 1$. The rough intuition for this 
equilibrium specification is that odd labeled players are relatively weak and even labeled players are relatively strong in the bargaining game $\Gamma^{\delta}\left(G_{s q+l i n e ~ 3}\right)$ for high $\delta .^{19}$

Odd labeled players are relatively weak and even labeled players are relatively strong in the bargaining games on the line of 3 and 5, and the square plus point networks, and players 2, 4,6 occupy the positions corresponding to even labels in these networks, in some isomorphic induced subnetworks following initial equilibrium agreements. The significant difference between the payoff of player 2 and the (almost identical) payoffs of players 4 and 6 is due to the initial equilibrium agreement between players 3 and 4, which undermines player 2's position. When 3 and 4 reach the first agreement, 2 is left in a bilateral bargaining game with 1 , which yields a limit payoff of $1 / 2$. This diminishes the "strong even position" effect in the three types of subnetworks enumerated above.

Similarly, player 1 is better off than player 3. Although 1 and 3 have symmetric positions in the network, 1 is at an advantage over 3 since initial equilibrium agreement obtains between 3 and 4 , but not between 1 and 4 . Player 7 is slightly weaker than player 3 because, as argued above, player 7's only neighbor, player 6, is significantly stronger than one of player 3's neighbors, player 2. Finally, player 5 is the weakest of all odd labelled players because his middle position is inferior to the peripheral positions of the other odd players in the subnetworks isomorphic to the line of 5 network induced by initial agreements across the links $(1,2)$ and $(2,3)$.

Players 1 and 4 are reluctant to reach an initial agreement in equilibrium because each can benefit from waiting to bargain with a weaker neighbor. It is possible for all other pairs of payers to reach initial agreements in equilibrium since no other two relatively strong players (according to the conjectured equilibrium) among the odd and even labeled players, respectively, are linked in the network. The conjectured initial agreement behavior is selfconfirming and leads to an MPE for high $\delta$.

Note that the positions of players 1 and 3 are symmetric in $G_{s q+l i n e}$, but their strategies in the MPE above are not identical. We can obtain another MPE for high $\delta$ by simply interchanging the roles of players 1 and 3 in the initial equilibrium agreement behavior considered above. These two pure strategy MPEs of $\Gamma^{\delta}\left(G_{s q+l i n e ~ 3}\right)$ for high $\delta$ are not payoff equivalent for players 1 and 3 .

\footnotetext{
${ }^{19}$ The words "weak" and "strong" vaguely mean payoffs significantly below and respectively above $1 / 2$.
} 
For sufficiently high $\delta$, a third MPE of $\Gamma^{\delta}\left(G_{s q+l i n e ~ 3}\right)$ exists, in which there is a common initial probability of agreement across the links $(1,4)$ and $(3,4)$ in the interval $(0,1) \cdot{ }^{20}$ The limit MPE initial agreement is $p$ with $p\left(G_{\text {sq+line } 3} ;(1,4)\right)=p\left(G_{\text {sq+line } 3} ;(3,4)\right) \approx{ }^{21} 0.528758$ and $p\left(G_{s q+l i n e ~} 3 ; i j\right)=1$ for all other links $i j$. The limit equilibrium payoff vector is $v$ with $v_{1}=v_{3} \approx 0.211, v_{2} \approx 0.755, v_{4} \approx 0.789, v_{5} \approx 0.068, v_{6} \approx 0.796, v_{7} \approx 0.170$. Therefore, for high $\delta$ the bargaining game $\Gamma^{\delta}\left(G_{s q+\text { line } 3}\right)$ has at least three MPEs. Note that the latter MPE is not payoff equivalent with either of the former two MPEs for any player.

\section{Conclusion}

Networks are ubiquitous in economic and social contexts, and have been the subject of extensive inquiry (Jackson (2008) offers an excellent overview). Nevertheless, there has been little analysis of models in which pairs of players connected by a network are matched to bargain over the division of surplus. Such models provide a natural framework to investigate the connection between network structure, feasible agreements, and the division of the gains from trade. The present paper and Manea (2008) represent initial steps in this direction.

In the model introduced here we establish existence of MPEs and show that MPEs are not necessarily unique. We relate the properties of MPEs to features of the underlying network. In many networks MPEs are incompatible with efficient matching even asymptotically as players become patient (or the time between matchings goes to zero). A major focus of the analysis is on constructing asymptotically efficient subgame perfect equilibria for every network. Our setting is perhaps the simplest in which the relevant issues can be addressed. In particular, we have assumed various symmetries in the matching technology and the potential gains from trade between connected agents. In future work we hope to relax some of these assumptions.

\footnotetext{
${ }^{20}$ The proof is similar to the one of Proposition 4.

${ }^{21}$ The exact limit initial MPE agreement probability common for the links $(1,4)$ and $(3,4)$ is one of the four roots of an irreducible polynomial of degree 4 .
} 


\section{Appendix A. Proof for Section 2}

Proof of Proposition 1. We first provide a characterization of MPE payoffs, and then use it to show that an MPE always exists. Fix $\delta \in(0,1)$. For a set of networks $\mathcal{H}$, a collection of Markov strategy profiles $(\sigma(H))_{H \in \mathcal{H}}$ for the respective games $\left(\Gamma^{\delta}(H)\right)_{H \in \mathcal{H}}$ is subgame consistent if for every pair of networks $H, H^{\prime} \in \mathcal{H}, \sigma(H)$ and $\sigma\left(H^{\prime}\right)$ induce the same behavior in any pair of identical subgames of $\Gamma^{\delta}(H)$ and $\Gamma^{\delta}\left(H^{\prime}\right)$. Formally, subgame consistency of $\left(\Gamma^{\delta}(H)\right)_{H \in \mathcal{H}}$ requires that $\sigma(H)\left(h_{t} ; i \rightarrow j\right)=\sigma\left(H^{\prime}\right)\left(h_{t^{\prime}}^{\prime} ; i \rightarrow j\right)$ and $\sigma(H)\left(h_{t} ; i \rightarrow j ; x\right)=\sigma\left(H^{\prime}\right)\left(h_{t^{\prime}}^{\prime} ; i \rightarrow j ; x\right)$ for all pairs of players $(i, j)$, all offers $x$, all $h_{t}$ and $h_{t^{\prime}}^{\prime}$ such that the players remaining in the subgame $h_{t}$ of $\Gamma^{\delta}(H)$ and the subgame $h_{t^{\prime}}^{\prime}$ of $\Gamma^{\delta}\left(H^{\prime}\right)$ induce identical networks (which include the link $i j$ ), and all $H, H^{\prime} \in \mathcal{H}$.

Suppose $\sigma^{* \delta}(G)$ is an MPE of $\Gamma^{\delta}(G)$. By the definition of an MPE, it must be that $\sigma^{* \delta}(G)$ belongs to a subgame consistent collection of MPEs $\left(\sigma^{* \delta}(\tilde{G})\right)_{\tilde{G} \in \mathcal{G}}$ of the respective games $\left(\Gamma^{\delta}(\tilde{G})\right)_{\tilde{G} \in \mathcal{G}}$ with corresponding payoffs $\left(v^{* \delta}(\tilde{G})\right)_{\tilde{G} \in \mathcal{G}}$. In particular, when $\Gamma^{\delta}(G)$ is played according to $\sigma^{* \delta}(G)$, every player $k$ has expected payoffs $v_{k}^{* \delta}(G)$ at the beginning of any subgame before which no agreement has occurred, and $v_{k}^{* \delta}(G \ominus\{i, j\})$ at the beginning of any subgame before which only $i$ and $j$ reached an agreement $(k \neq i, j)$.

Fix a history $\left(h_{t} ; i \rightarrow j\right)$ along which no agreement has been reached $\left(G\left(h_{t}\right)=G\right)$. In the subgame following $\left(h_{t} ; i \rightarrow j\right)$, it must be that the strategy $\sigma_{j}^{* \delta}(G)$ specifies that player $j$ accept any offer larger than $\delta v_{j}^{* \delta}(G)$, and reject any offer smaller than $\delta v_{j}^{* \delta}(G)$. Then it is not optimal for $i$ to make an offer $x>\delta v_{j}^{* \delta}(G)$ to $j$, since $i$ would be better off making some offer in the interval $\left(\delta v_{j}^{* \delta}(G), x\right)$ instead, as $j$ accepts such offers with probability 1. Hence, in equilibrium $i$ has to offer $j$ at most $\delta v_{j}^{* \delta}(G)$ with probability 1 , and $j$ may accept with positive probability only offers of $\delta v_{j}^{* \delta}(G)$. Let $p$ be the probability (conditional on $\left.\left(h_{t} ; i \rightarrow j\right)\right)$ of the joint event that $i$ offers $\delta v_{j}^{* \delta}(G)$ to $j$ and the offer is accepted. The payoff of any player $k \neq i, j$ at the beginning of the next period is $v_{k}^{* \delta}(G \ominus\{i, j\})$ in case $i$ and $j$ reach an agreement, and $v_{k}^{* \delta}(G)$ otherwise. Therefore, the time $t$ expected payoff of $k$ conditional on the history $\left(h_{t} ; i \rightarrow j\right)$ is $p \delta v_{k}^{* \delta}(G \ominus\{i, j\})+(1-p) \delta v_{k}^{* \delta}(G)$.

If $\delta\left(v_{i}^{* \delta}(G)+v_{j}^{* \delta}(G)\right)<1$, when $i$ is chosen to propose to $j$, it must be that in equilibrium $i$ offers $\delta v_{j}^{* \delta}(G)$ and agreement obtains with probability 1, i.e., $p=1$. For, if $p<1$ then $i$ 's expected payoff conditional on offering $\delta v_{j}^{* \delta}(G)$ is $p\left(1-\delta v_{j}^{* \delta}(G)\right)+(1-p) \delta v_{i}^{* \delta}(G)<1-\delta v_{j}^{* \delta}(G)$, 
while conditional on offering $\delta v_{j}^{* \delta}(G)+\varepsilon(\varepsilon>0)$ is $1-\delta v_{j}^{* \delta}(G)-\varepsilon$ (we argued that $j$ accepts offers greater than $\delta v_{j}^{* \delta}(G)$ with probability 1$)$. But for small $\varepsilon>0, p\left(1-\delta v_{j}^{* \delta}(G)\right)+(1-$ p) $\delta v_{i}^{* \delta}(G)<1-\delta v_{j}^{* \delta}(G)-\varepsilon$. Hence it is not optimal for $i$ to offer $\delta v_{j}^{* \delta}(G)$ to $j$. By the same token, offers smaller than $\delta v_{j}^{* \delta}(G)$ are not optimal for $i$ since they are rejected with probability 1 and yield expected payoff $\delta v_{i}^{* \delta}(G)<1-\delta v_{j}^{* \delta}(G)-\varepsilon$. We already argued that no offer greater than $\delta v_{j}^{* \delta}(G)$ may be optimal for $i$ either. Therefore, if $\delta\left(v_{i}^{* \delta}(G)+v_{j}^{* \delta}(G)\right)<1$ and $p<1$, then $i$ cannot have a best response to $j$ 's equilibrium strategy. We established that if $\delta\left(v_{i}^{* \delta}(G)+v_{j}^{* \delta}(G)\right)<1$ then $p=1$. Similarly, if $\delta\left(v_{i}^{* \delta}(G)+v_{j}^{* \delta}(G)\right)>1$ then $p=0$. If $\delta\left(v_{i}^{* \delta}(G)+v_{j}^{* \delta}(G)\right)=1$ then $p$ can be any number in the interval $[0,1]$.

Consider the correspondence $f^{i \rightarrow j}:[0,1]^{n} \rightrightarrows[0,1]^{n}$ defined by

$$
\begin{aligned}
f^{i \rightarrow j}(v)=\left\{p \delta(\underbrace{v^{* \delta}(G \ominus\{i, j\})}_{-i,-j}, \underbrace{1-\delta v_{j}}_{i}, \underbrace{\delta v_{j}}_{j})+(1-p) \delta v \mid\right. \\
\left.p=1(0) \text { if } \delta\left(v_{i}+v_{j}\right)<(>) 1 \text {, and } p \in[0,1] \text { if } \delta\left(v_{i}+v_{j}\right)=1\right\},
\end{aligned}
$$

where $(\underbrace{v^{* \delta}(G \ominus\{i, j\})}_{-i,-j}, \underbrace{1-\delta v_{j}}_{i}, \underbrace{\delta v_{j}}_{j})$ represents the vector in $[0,1]^{n}$ with the $k(\neq i, j)$ coordinate equal to $v_{k}^{* \delta}(G \ominus\{i, j\}), i$ coordinate equal to $1-\delta v_{j}$ and $j$ coordinate equal to $\delta v_{j}$. Note that $f_{k}^{i \rightarrow j}\left(v^{* \delta}(G)\right)$ is the set of possible time $t$ expected payoffs for player $k$ conditional on the history $\left(h_{t} ; i \rightarrow j\right)$, where the behaviors of $i$ and $j$ are constrained by the equilibrium analysis above. In other words, $f^{i \rightarrow j}\left(v^{* \delta}(G)\right)$ computes the time $t$ expected payoff vectors conditional on the history $\left(h_{t} ; i \rightarrow j\right)$ that are not rejected by our partial equilibrium analysis when players behave according to $\sigma^{* \delta}(G)$.

Let $f:[0,1]^{n} \rightrightarrows[0,1]^{n}$ be the correspondence defined by

$$
f(v)=\sum_{\{i \rightarrow j \mid i j \in G\}} \frac{1}{2 e^{G}} f^{i \rightarrow j}(v)
$$

(recall that $e^{G}$ denotes the total number of links in $G$ ). Let $h_{t}$ be a history along which no agreement has occurred, and consider the resulting period $t$ subgame. Since nature selects player $i$ to make an offer to player $j$ with probability $1 /\left(2 e^{G}\right)$ for each link $i j \in G$, and conditional on the selection, $f^{i \rightarrow j}\left(v^{* \delta}(G)\right)$ describes the time $t$ expected payoffs constrained by the equilibrium requirements, $f\left(v^{* \delta}(G)\right)$ is the set of expected payoffs at the beginning of the subgame $h_{t}$ that are not rejected by our partial equilibrium analysis when players 
behave according to $\sigma^{* \delta}(G)$. In equilibrium, the time $t$ expected payoff vector conditional on the history $h_{t}$ is $v^{* \delta}(G)$, hence $v^{* \delta}(G) \in f\left(v^{* \delta}(G)\right)$. Therefore, $v^{* \delta}(G)$ is a fixed point of $f$. Conversely, any fixed point of $f$ is an MPE payoff vector.

Lemma 6. A vector $v$ is a Markov perfect equilibrium payoff of $\Gamma^{\delta}(G)$ if and only if there exists a subgame consistent collection of Markov perfect equilibria of the games $\left(\Gamma^{\delta}(\tilde{G})\right)_{\tilde{G} \in \mathcal{G}^{0}}$ with respective payoffs $\left(v^{* \delta}(\tilde{G})\right)_{\tilde{G} \in \mathcal{G}^{0}}$ such that $v$ is a fixed point of the correspondence $f$ defined by A.1-A.2. ${ }^{22}$

Proof. We already proved the "only if" part. To prove the "if" part, suppose that the subgame consistent collection of MPEs $\left(\sigma^{* \delta}(\tilde{G})\right)_{\tilde{G} \in \mathcal{G}^{0}}$ of the games $\left(\Gamma^{\delta}(\tilde{G})\right)_{\tilde{G} \in \mathcal{G}^{0}}$ with respective payoffs $\left(v^{* \delta}(\tilde{G})\right)_{\tilde{G} \in \mathcal{G}^{0}}$ defines the correspondence $f$ by A.1-A.2, and that $v \in f(v)$. It follows that

$$
v=\sum_{\{i \rightarrow j \mid i j \in G\}} \frac{1}{2 e} z^{i \rightarrow j},
$$

where $z^{i \rightarrow j} \in f^{i \rightarrow j}(v)$. Then, there exists $p^{i \rightarrow j}$ such that

$$
z^{i \rightarrow j}=p^{i \rightarrow j} \delta(\underbrace{v^{* \delta}(G \ominus\{i, j\})}_{-i,-j}, \underbrace{1-\delta v_{j}}_{i}, \underbrace{\delta v_{j}}_{j})+\left(1-p^{i \rightarrow j}\right) \delta v,
$$

with $p^{i \rightarrow j}=1(0)$ if $\delta\left(v_{i}+v_{j}\right)<(>) 1$ and $p^{i \rightarrow j} \in[0,1]$ if $\delta\left(v_{i}+v_{j}\right)=1$.

The strategy profile $\sigma^{* \delta}(G)$ (implicitly) defined below constitutes an MPE with payoffs $v$. We first define the strategies for histories $h_{t}$ along which at least one agreement occurred. Recall that $G\left(h_{t}\right)$ denotes the network induced by the players remaining in the subgame $h_{t}$. Construct the time $t$ strategy of each player according to the time 0 behavior specified by $\sigma^{* \delta}\left(G\left(h_{t}\right)\right) .{ }^{23}$ For histories along which no agreement has occurred, $\sigma^{* \delta}(G)$ specifies that when $i$ is chosen to propose to $j$ he offers $\min \left(1-\delta v_{i}, \delta v_{j}\right)$, and when $i$ has to respond to an offer from $j$ he accepts with probability 1 any offer greater than $\delta v_{i}$, accepts with probability $p^{i \rightarrow j}$ an offer of $\delta v_{i}$, and rejects with probability 1 any smaller offers. ${ }^{24}$

\footnotetext{
${ }^{22}$ Recall that $\mathcal{G}^{0}$ denotes the set of subnetworks of $G$, different from $G$, induced by the players remaining in any subgame of $\Gamma^{\delta}(G)$.

${ }^{23}$ Formally, $\sigma_{i}^{* \delta}(G)\left(h_{t} ; i \rightarrow j\right)=\sigma_{i}^{* \delta}\left(G\left(h_{t}\right)\right)\left(h_{0} ; i \rightarrow j\right)$ and $\sigma_{i}^{* \delta}(G)\left(h_{t} ; j \rightarrow i ; x\right)=\sigma_{i}^{* \delta}\left(G\left(h_{t}\right)\right)\left(h_{0} ; j \rightarrow i ; x\right)$ for all $i j \in G\left(h_{t}\right)$ and $x \in[0,1]$, where $h_{0}$ denotes an empty history.

${ }^{24}$ Payoff irrelevant MPE multiplicity may arise for two reasons. First, if $\delta\left(v_{i}+v_{j}\right)>1$, when $i$ is selected to propose to $j$, in the construction above $i$ offers $\min \left(1-\delta v_{i}, \delta v_{j}\right)=1-\delta v_{i}$ to $j$ and the offer is rejected. The equilibrium construction may be modified so that $i$ offers $j$ any (mixed) offer $x<\delta v_{j}$, as rejection obtains regardless (if $j$ rejects offers of $\delta v_{j}$ with probability 1 the constraint is $x \leq \delta v_{j}$ ). Second, if $\delta\left(v_{i}+v_{j}\right)=1$,
} 
The subgame consistency of the collection $\left(\sigma^{* \delta}(\tilde{G})\right)_{\tilde{G} \in \mathcal{G}^{0}}$ guarantees that under the constructed $\sigma^{* \delta}(G)$ the expected payoffs in any subgame of $\Gamma^{\delta}(G)$ with induced network $\tilde{G} \in \mathcal{G}^{0}$ are $v^{* \delta}(\tilde{G})$, and that $\sigma^{* \delta}(G)$ is an MPE with expected payoffs $v$.

We can now use Lemma 6 to show existence of MPEs. We prove more generally that there exists a subgame consistent collection of MPEs for the games $\left(\Gamma^{\delta}(\tilde{G})\right)_{\tilde{G} \in \mathcal{G}\left(n^{\prime}\right)}$, where $\mathcal{G}\left(n^{\prime}\right)$ denotes the subset of subnetworks in $\mathcal{G}$ that have at most $n^{\prime}$ vertices. We proceed by induction on $n^{\prime}$. For $n^{\prime}=0,1$, the statement is trivially satisfied since the corresponding games are eventless.

Suppose we proved the statement for all lower values, and we proceed to proving it for $n^{\prime}$ $\left(2 \leq n^{\prime} \leq n\right)$. By the induction hypothesis, there exists a subgame consistent collection of MPEs $\left(\sigma^{* \delta}(\tilde{G})\right)_{\tilde{G} \in \mathcal{G}\left(n^{\prime}-1\right)}$ of the corresponding games $\left(\Gamma^{\delta}(\tilde{G})\right)_{\tilde{G} \in \mathcal{G}\left(n^{\prime}-1\right)}$. Fix a network $G^{\prime} \in \mathcal{G}$ with $n^{\prime}$ vertices, and let $\mathcal{G}^{\prime 0}$ be the set of all subnetworks of $G^{\prime}$, excluding $G^{\prime}$, induced in all subgames of $\Gamma^{\delta}\left(G^{\prime}\right)$. Then $\mathcal{G}^{\prime 0}$ is a subset of $\mathcal{G}\left(n^{\prime}-1\right)$. Therefore, the collection of MPEs $\left(\sigma^{* \delta}(\tilde{G})\right)_{\tilde{G} \in \mathcal{G}^{\prime 0}}$ for the games $\left(\Gamma^{\delta}(\tilde{G})\right)_{G \in \mathcal{G}^{\prime 0}}$ is subgame consistent, and we can use their payoffs to define $f^{i \rightarrow j}$ and $f$ as in A.1-A.2 for the game $\Gamma^{\delta}\left(G^{\prime}\right)$.

Note that each $f^{i \rightarrow j}:[0,1]^{n} \rightarrow[0,1]^{n}$ is an upper hemi-continuous correspondence with non-empty convex images. The correspondence $f:[0,1]^{n} \rightarrow[0,1]^{n}$ is a convex combination of the correspondences $\left(f^{i \rightarrow j}\right)_{\left\{i \rightarrow j \mid i j \in G^{\prime}\right\}}$, hence it is upper hemi-continuous with non-empty convex images as well. By Kakutani's fixed point theorem, $f$ has a fixed point.

We can use the steps from Lemma 6 to construct an MPE $\sigma^{* \delta}\left(G^{\prime}\right)$ of $\Gamma^{\delta}\left(G^{\prime}\right)$ so that the collection of MPEs $\left(\sigma^{* \delta}(\tilde{G})\right)_{\tilde{G} \in \mathcal{G}\left(n^{\prime}-1\right) \cup\left\{G^{\prime}\right\}}$ is subgame consistent. If we append the MPEs $\sigma^{* \delta}\left(G^{\prime}\right)$ for all subnetworks $G^{\prime} \in \mathcal{G}$ with $n^{\prime}$ vertices to the subgame consistent collection of MPEs $\left(\sigma^{* \delta}(\tilde{G})\right)_{\tilde{G} \in \mathcal{G}\left(n^{\prime}-1\right)}$, the resulting collection of MPEs $\left(\sigma^{* \delta}(\tilde{G})\right)_{\tilde{G} \in \mathcal{G}\left(n^{\prime}\right)}$ for the respective games $\left(\Gamma^{\delta}(\tilde{G})\right)_{\tilde{G} \in \mathcal{G}\left(n^{\prime}\right)}$ is subgame consistent. This completes the proof of the induction step.

in the construction above $i$ 's offer to $j$ is accepted with probability $p^{i \rightarrow j}$, and $j$ 's offer to $i$ is accepted with probability $p^{j \rightarrow i}$. If $p^{i \rightarrow j}+p^{j \rightarrow i} \neq 0,2$ then the equilibrium construction may be modified so that the two agreement probabilities become $p^{i \rightarrow j}+\varepsilon$ and $p^{j \rightarrow i}-\varepsilon$, respectively, for a range of values of $\varepsilon$. 


\section{Appendix B. Proofs for Section 3}

Proof of Lemma 1. We proceed by contradiction. Suppose that $\tilde{\tilde{M}}$ is an efficient match of $\tilde{\tilde{G}}$ such that $p^{*}(\tilde{G} ; l m)<1, \forall l m \in \tilde{\tilde{M}}$. Then it must be that for sufficiently large $\alpha$,

$$
\delta_{\alpha}\left(v_{l}^{* \delta_{\alpha}}(\tilde{G})+v_{m}^{* \delta_{\alpha}}(\tilde{G})\right) \geq 1, \forall l m \in \tilde{\tilde{M}} .
$$

Fixing such an $\alpha$, when we add up the inequalities above across all links in $\tilde{\tilde{M}}$ we obtain that

$$
\sum_{l m \in \tilde{\tilde{M}}} \delta_{\alpha}\left(v_{l}^{* \delta_{\alpha}}(\tilde{G})+v_{m}^{* \delta_{\alpha}}(\tilde{G})\right) \geq \mu(\tilde{\tilde{M}})
$$

As $\tilde{\tilde{N}}$ is $\tilde{G}$-efficiently closed, it follows that players in $\tilde{\tilde{N}}$ can only reach agreements with players in $\tilde{\tilde{N}}$ under $\bar{\sigma}^{* \delta_{\alpha}}(\tilde{G})$. Since every player in $\tilde{\tilde{N}}$ can only be involved in one agreement, and each agreement yields a unit total surplus, it must be that

$$
\sum_{k \in \tilde{\tilde{N}}} v_{k}^{* \delta_{\alpha}}(\tilde{G}) \leq \mu(\tilde{\tilde{M}})
$$

Therefore,

$$
\sum_{k \in \tilde{\tilde{N}}} v_{k}^{* \delta_{\alpha}}(\tilde{G}) \leq \mu(\tilde{\tilde{M}}) \leq \sum_{l m \in \tilde{\tilde{M}}} \delta_{\alpha}\left(v_{l}^{* \delta_{\alpha}}(\tilde{G})+v_{m}^{* \delta_{\alpha}}(\tilde{G})\right) \leq \sum_{k \in \tilde{\tilde{N}}} \delta_{\alpha} v_{k}^{* \delta_{\alpha}}(\tilde{G}) .
$$

Since $\delta_{\alpha} \in(0,1)$, we need that $\sum_{\tilde{\tilde{N}} \in \tilde{\tilde{N}}} v_{k}^{* \delta_{\alpha}}(\tilde{G})=\mu(\tilde{\tilde{M}})=0$. However, $\mu(\tilde{\tilde{M}}) \geq 1$, because $\tilde{\tilde{G}}$ has a non-empty set of links and $\tilde{\tilde{M}}$ is an efficient match of $\tilde{\tilde{G}}$. This yields a contradiction.

Lemma 7 below, on which most of the preliminary results hinge, necessitates a review of the Gallai-Edmonds decomposition theorem [13]. This is a graph theoretical result concerning the structure of efficient matchings. The following partition of the set of vertices $\tilde{N}$ of the network $\tilde{G}$ is essential for the result. The set of players under-demanded in $\tilde{G}$, denoted $U(\tilde{G})$, consists of the players that are not always efficiently matched in $\tilde{G}$. The set of players over-demanded in $\tilde{G}$, denoted $O(\tilde{G})$, consists of the players that are connected to at least one underdemanded player. The set of players perfectly matched in $\tilde{G}$, denoted $P(\tilde{G})$, 
consists of the players that are not under-demanded or over-demanded in $\tilde{G} \cdot{ }^{25}$ Formally,

$$
\begin{aligned}
& U(\tilde{G})=\{u \mid \exists \text { efficient match } \tilde{M} \text { of } \tilde{G}, u \notin \tilde{M}\} \\
& O(\tilde{G})=\{o \mid \exists u \in U(\tilde{G}), u o \in \tilde{G}\} \\
& P(\tilde{G})=\tilde{N} \backslash(U(\tilde{G}) \cup O(\tilde{G})) .
\end{aligned}
$$

We only state the contents of the Gallai-Edmonds decomposition theorem necessary for our proofs.

Theorem 2 (Gallai-Edmonds). For every efficient match $\tilde{M}$ of $\tilde{G}$, for every o $\in O(\tilde{G})$ there exists $u \in U(\tilde{G})$ such that $u o \in \tilde{M}$. The sets $P(\tilde{G})$ and $U(\tilde{G}) \cup O(\tilde{G})$ are $\tilde{G}$-efficiently closed.

Denote by $\hat{U}(\tilde{G})$ the set of players in $U(\tilde{G})$ that are not isolated in $\tilde{G}$. Thus $\hat{U}(\tilde{G})=\emptyset$ $(\hat{U}(\tilde{G}) \neq \emptyset)$ means that in $\tilde{G}$ (not) all non-isolated players are always efficiently matched.

Lemma 7. Suppose that $\hat{U}(\tilde{G}) \neq \emptyset$, and that $v_{l}^{*}(\tilde{G})+v_{m}^{*}(\tilde{G}) \geq 1$ for all $\tilde{G}$-efficient links $l m$. Then there exist $l, m \in U(\tilde{G}) \cup\left\{g \mid v_{g}^{*}(\tilde{G})=1\right\}$ such that $p^{*}(\tilde{G} ; l m)=1$.

Proof of Lemma 7. Let $\tilde{M}$ be an efficient match of $\tilde{G}$. By hypothesis,

$$
v_{l}^{*}(\tilde{G})+v_{m}^{*}(\tilde{G}) \geq 1, \forall l m \in \tilde{M} .
$$

Adding up the inequalities B.1 across all links in $\tilde{M}$, we obtain that

$$
\sum_{l m \in \tilde{M}} v_{l}^{*}(\tilde{G})+v_{m}^{*}(\tilde{G}) \geq \mu(\tilde{G}) .
$$

However, note that the constraints of the production technology imply that

$$
\sum_{k \in \tilde{G}} v_{k}^{*}(\tilde{G}) \leq \mu(\tilde{G}) .
$$

Inequalities B.1-B.3 can be satisfied only if they all hold with equality. Therefore,

$$
\begin{aligned}
v_{l}^{*}(\tilde{G})+v_{m}^{*}(\tilde{G}) & =1, \forall l m \in \tilde{M} \\
v_{u}^{*}(\tilde{G}) & =0, \forall u \notin \tilde{M} .
\end{aligned}
$$

The argument above shows that $v_{l}^{*}(\tilde{G})+v_{m}^{*}(\tilde{G})=1$ for every $\tilde{G}$-efficient link $\operatorname{lm}$ (by definition, every $\tilde{G}$-efficient link is part of an efficient match of $\tilde{G})$, and $v_{u}^{*}(\tilde{G})=0$ for every ${ }^{25}$ The terms under-demanded, over-demanded, and perfectly matched were coined in [3] and [18]. 
$u \in U(\tilde{G})$ (by definition, for every $u \in U(\tilde{G})$ there exists an efficient match of $\tilde{G}$ that does not cover $u)$.

Fix $o \in O(\tilde{G})$, and let $\tilde{M}$ be an efficient match of $\tilde{G}$. By the Gallai-Edmonds decomposition theorem, there is a $u \in U(\tilde{G})$ such that $u o \in \tilde{M}$. As argued above, $v_{u}^{*}(\tilde{G})=0$ and $v_{u}^{*}(\tilde{G})+$ $v_{o}^{*}(\tilde{G})=1$. Hence $v_{o}^{*}(\tilde{G})=1$. We established that $O(\tilde{G}) \subset\left\{g \mid v_{g}^{*}(\tilde{G})=1\right\}$.

By the Gallai-Edmonds decomposition theorem, $U(\tilde{G}) \cup O(\tilde{G})$ is $\tilde{G}$-efficiently closed. Then Lemma 1 implies that there exist $l, m \in U(\tilde{G}) \cup O(\tilde{G}) \subset U(\tilde{G}) \cup\left\{g \mid v_{g}^{*}(\tilde{G})=1\right\}$ such that $p^{*}(\tilde{G} ; l m)=1$.

Proof of Proposition 3. If for some network $\tilde{G} \in \mathcal{G}$ with $\hat{U}(\tilde{G})=\emptyset, v_{i}^{*}(\tilde{G}) \geq 1 / 2$ for all $i$ that are always efficiently matched in $\tilde{G}$, then $v_{i}^{*}(\tilde{G})=1 / 2$ for all such $i$. Indeed, this is a consequence of the production technology constraint $\sum_{i \in \tilde{G}} v_{i}^{* \delta_{\alpha}}(\tilde{G}) \leq \mu(\tilde{G}), \forall \alpha \geq 0$, which in the limit as $\alpha \rightarrow \infty$ becomes $\sum_{i \in \tilde{G}} v_{i}^{*}(\tilde{G}) \leq \mu(\tilde{G})$. Hence it suffices to prove the first part of the proposition.

For a contradiction, let $\tilde{G}$ be a counterexample to the first part of the proposition with the least number of vertices. Then there is a player $i$ in $\tilde{G}$ that is always efficiently matched in $\tilde{G}$ with $v_{i}^{*}(\tilde{G})<1 / 2$. Suppose first that $\tilde{G}$ is not a non-trivial perfect network. We reach a contradiction in 8 steps.

Step 1. $v_{g}^{*}(\tilde{G} \ominus\{l, m\}) \geq 1 / 2$ for all $g \neq l, m$ always efficiently matched in $\tilde{G}$ and for every $\tilde{G}$-efficient $l m$

By the minimality of the counterexample $\tilde{G}$, for every player $g$ that is always efficiently matched in $\tilde{G} \ominus\{l, m\}$, we have that $v_{g}^{*}(\tilde{G} \ominus\{l, m\}) \geq 1 / 2$ for any $\tilde{G}$-efficient link $l m$. To reach the conclusion, note that for any $\tilde{G}$-efficient link $l m$, a player that is always efficiently matched in $\tilde{G}$ is also always efficiently matched in $\tilde{G} \ominus\{l, m\}$.

Step 2. $p^{*}(\tilde{G} ; l m)=0$ for all $l, m \neq i$

For a contradiction, assume that $p^{*}(\tilde{G} ; l m)>0$ for some $\tilde{G}$-efficient link $l m$ with $l, m \neq i$. A deviation by $i$ from $\bar{\sigma}_{i}^{* \delta_{\alpha}}(\tilde{G})$ to behavior that avoids any agreements before such links $l m$ are selected for bargaining, yields the following equilibrium requirement,

$$
v_{i}^{* \delta_{\alpha}}(\tilde{G}) \geq \sum_{l m} \frac{1}{e^{\tilde{G}}} p^{* \delta_{\alpha}}(\tilde{G} ; l m) \delta_{\alpha} v_{i}^{* \delta_{\alpha}}(\tilde{G} \ominus\{l, m\})+\left(1-\sum_{l m} \frac{1}{e^{\tilde{G}}} p^{* \delta_{\alpha}}(\tilde{G} ; l m)\right) \delta_{\alpha} v_{i}^{* \delta_{\alpha}}(\tilde{G}),
$$


where summations are over the set $\{l m \mid l m$ is $\tilde{G}$-efficient, with $l, m \neq i\}$. Taking the limit $\alpha \rightarrow \infty$, since $v_{i}^{*}(\tilde{G} \ominus\{l, m\}) \geq 1 / 2$ for all $\tilde{G}$-efficient links $l m$ (Step 1$)$ and $\sum_{l m} \frac{1}{e^{\tilde{G}}} p^{*}(\tilde{G} ; l m)>$ 0 , we obtain that $v_{i}^{*}(\tilde{G}) \geq 1 / 2$, a contradiction.

Step 3. $p^{*}(\tilde{G} ; i j)=1$ for all $j$ such that $i j$ is $\tilde{G}$-efficient

Fix a $\tilde{G}$-efficient link $i j$ for player $i$. By definition, there exists an efficient match $\tilde{M}$ of $\tilde{G}$ that includes the link $i j$. Since $p^{*}(\tilde{G} ; l m)=0$ for all $l m \in \tilde{M} \ominus\{i, j\}$ (Step 2), Corollary 1 implies that $p^{*}(\tilde{G} ; i j)=1$.

Step 4. $\exists j \neq k$ (fixed for the rest of the proof) such that $i j$ and $i k$ are $\tilde{G}$-efficient

Since $i$ is always efficiently matched in $\tilde{G}$, there exists $j$ such that $i j$ is $\tilde{G}$-efficient. If there exists no $k \neq j$ such that $i k$ is also $\tilde{G}$-efficient, then it must be that $i j$ is player $i$ 's unique $\tilde{G}$-efficient link, hence $i$ and $j$ are only efficiently matched to each other in $\tilde{G}$. Then players $i$ and $j$ only bargain with each other in every subgame of $\bar{\Gamma}^{\delta}(\tilde{G})$. It can be easily argued that $v_{i}^{* \delta_{\alpha}}(\tilde{G})=v_{j}^{* \delta_{\alpha}}(\tilde{G})$. Since $p^{*}(\tilde{G} ; i j)=1$ (Step 3), we need that $v_{i}^{*}(\tilde{G})+v_{j}^{*}(\tilde{G})=1$. Therefore, $v_{i}^{*}(\tilde{G})=1 / 2$, a contradiction.

Step 5. $v_{g}^{*}(\tilde{G}) \geq 1 / 2$ for every $g \neq i$ that is always efficiently matched in $\tilde{G}$

Fix $g \neq i$ that is always efficiently matched in $\tilde{G}$. At least one of the links $i j$ and $i k$ (from Step 4) does not involve $g$, and $p^{*}(\tilde{G} ; i j)=p^{*}(\tilde{G} ; i k)=1 \neq 0$ (Step 3). The conclusion follows by an argument analogous to Step 2, with $i$ replaced by $g$.

Step 6. $v_{i}^{*}(\tilde{G})+v_{g}^{*}(\tilde{G}) \geq 1$ for all $g$ such that $i g$ is $\tilde{G}$-efficient

Fix a player $g$ such that $i g$ is a $\tilde{G}$-efficient link. Since the probability that $i$ is involved in all initial agreements when $\bar{\Gamma}^{\delta_{\alpha}}(\tilde{G})$ is played according to $\bar{\sigma}^{* \delta_{\alpha}}(\tilde{G})$ converges to 1 as $\alpha \rightarrow \infty$, a deviation by $i$ from $\bar{\sigma}_{i}^{* \delta_{\alpha}}(\tilde{G})$ to offering (slightly more than) $\delta_{\alpha} v_{g}^{* \delta_{\alpha}}(\tilde{G})$ to $g$ and avoiding any other agreements, yields the following equilibrium requirement,

$$
v_{i}^{* \delta_{\alpha}}(\tilde{G}) \geq \frac{1}{2 e^{\tilde{G}}}\left(1-\delta_{\alpha} v_{g}^{* \delta_{\alpha}}(\tilde{G})\right)+\frac{2 e^{\tilde{G}}-1}{2 e^{\tilde{G}}} \delta_{\alpha} v_{i}^{* \delta_{\alpha}}(\tilde{G})+\nu_{\alpha},
$$

where $\nu_{\alpha}$ converges to 0 as $\alpha \rightarrow \infty$, corresponding to $i$ 's expected payoff conditional on the vanishing probability event that an agreement that does not involve $i$ occurs (Step 2). Taking the limit $\alpha \rightarrow \infty$, we obtain that $v_{i}^{*}(\tilde{G})+v_{g}^{*}(\tilde{G}) \geq 1$.

Step 7. $\hat{U}(\tilde{G})=\emptyset$ leads to a contradiction 
Suppose that $\hat{U}(\tilde{G})=\emptyset$. Let $i g$ be a $\tilde{G}$-efficient link with $g \neq j$. Then $\hat{U}(\tilde{G} \ominus\{i, g\})=\emptyset$. By Step $1, v_{h}^{*}(\tilde{G} \ominus\{i, g\}) \geq 1 / 2$ for all $h$ that are always efficiently matched in $\tilde{G} \ominus\{i, g\}$. Then by the argument in the first paragraph of the proof, $v_{h}^{*}(\tilde{G} \ominus\{i, g\})=1 / 2$ for all such $h$. Since $j$ is non-isolated in $\tilde{G}(i j \in \tilde{G})$ and $\hat{U}(\tilde{G})=\emptyset, j$ is always efficiently matched in $\tilde{G}$, and also in $\tilde{G} \ominus\{i, g\}$. Hence, $v_{j}^{*}(\tilde{G} \ominus\{i, g\})=1 / 2$.

By definition,

$$
\begin{array}{r}
v_{j}^{* \delta_{\alpha}}(\tilde{G})=\sum_{g} \frac{1}{e^{\tilde{G}}} p^{* \delta_{\alpha}}(\tilde{G} ; i g) \delta_{\alpha} v_{j}^{* \delta_{\alpha}}(\tilde{G} \ominus\{i, g\})+\frac{1}{2 e^{\tilde{G}}}\left(1-\delta_{\alpha} v_{i}^{* \delta_{\alpha}}(\tilde{G})\right) \\
+\left(1-\sum_{g} \frac{1}{e^{\tilde{G}}} p^{* \delta_{\alpha}}(\tilde{G} ; i g)-\frac{1}{2 e^{\tilde{G}}}\right) \delta_{\alpha} v_{j}^{* \delta_{\alpha}}(\tilde{G})+\nu_{\alpha}^{\prime}
\end{array}
$$

where summations are over the set $\{g \mid i g$ is $\tilde{G}$-efficient, $g \neq j\}$, and $\nu_{\alpha}^{\prime}$ converges to 0 as $\alpha \rightarrow \infty$, corresponding to $j$ 's expected payoff conditional on the vanishing probability events that an agreement that does not involve $i$ occurs or that $i$ and $j$ do not reach an agreement when matched to bargain (Steps 2 and 3). Taking the limit $\alpha \rightarrow \infty$, we obtain that

$$
v_{j}^{*}(\tilde{G})=\pi \frac{1}{2}+\frac{1}{2 e^{\tilde{G}}}\left(1-v_{i}^{*}(\tilde{G})\right)+\left(1-\pi-\frac{1}{2 e^{\tilde{G}}}\right) v_{j}^{*}(\tilde{G})
$$

where $\pi=\sum_{g} \frac{1}{e^{\tilde{G}}} p^{*}(\tilde{G} ; i g) \geq \frac{1}{e^{\tilde{G}}} p^{*}(\tilde{G} ; i k)>0$. By Step $6,1-v_{i}^{*}(\tilde{G}) \leq v_{j}^{*}(\tilde{G})$, hence

$$
\left(\pi+\frac{1}{2 e^{\tilde{G}}}\right)\left(1-v_{i}^{*}(\tilde{G})\right) \leq\left(\pi+\frac{1}{2 e^{\tilde{G}}}\right) v_{j}^{*}(\tilde{G})=\pi \frac{1}{2}+\frac{1}{2 e^{\tilde{G}}}\left(1-v_{i}^{*}(\tilde{G})\right),
$$

which leads to $v_{i}^{*}(\tilde{G}) \geq 1 / 2($ as $\pi>0)$, a contradiction.

Step 8. $\hat{U}(\tilde{G}) \neq \emptyset$ leads to a contradiction

Suppose that $\hat{U}(\tilde{G}) \neq \emptyset$. By Step 6 ,

$$
v_{l}^{*}(\tilde{G})+v_{m}^{*}(\tilde{G}) \geq 1 \text { for all } \tilde{G} \text {-efficient } l m \text { with } i \in\{l, m\} .
$$

Step 2 established that $p^{*}(\tilde{G} ; l m)=0$ for all $l, m \neq i$, hence

$$
v_{l}^{*}(\tilde{G})+v_{m}^{*}(\tilde{G}) \geq 1 \text { for all } \tilde{G} \text {-efficient } l m \text { with } i \notin\{l, m\} .
$$

Thus all hypotheses of Lemma 7 are satisfied. Then there exist $l, m \in U(\tilde{G}) \cup\left\{g \mid v_{g}^{*}(\tilde{G})=1\right\}$ such that $p^{*}(\tilde{G} ; l m)=1$. By assumption, $i \notin U(\tilde{G})$ and $v_{i}^{*}(\tilde{G})<1 / 2<1$, hence $l, m \neq i$. This is a contradiction with Step 2. 
Now suppose that $\tilde{G}$ is a non-trivial perfect network. By an argument similar to Step 5, it follows that $v_{g}^{*}(\tilde{G}) \geq 1 / 2$ for all $g \notin\{h(\tilde{G}), k(\tilde{G})\}$. Assume without loss of generality that $v_{k(\tilde{G})}^{*}(\tilde{G})<1 / 2$.

A deviation by $k(\tilde{G})$ from $\bar{\sigma}_{k(\tilde{G})}^{* \delta_{\alpha}}(\tilde{G})$ to behavior that avoids any agreement, except for the case in which $k(\tilde{G})$ is selected to make an offer to $j(\tilde{G})$, where $k(\tilde{G})$ offers (slightly more than) $\delta_{\alpha} v_{j(\tilde{G})}^{* \delta_{\alpha}}(\tilde{G})$, yields the following equilibrium requirement,

$$
\begin{array}{r}
v_{k(\tilde{G})}^{* \delta_{\alpha}}(\tilde{G}) \geq \sum_{l m} \frac{1}{e^{\tilde{G}}} p^{* \delta_{\alpha}}(\tilde{G} ; \operatorname{lm}) \delta_{\alpha} v_{k(\tilde{G})}^{* \delta_{\alpha}}(\tilde{G} \ominus\{l, m\})+\frac{1}{2 e^{\tilde{G}}}\left(1-\delta_{\alpha} v_{j(\tilde{G})}^{* \delta_{\alpha}}(\tilde{G})\right) \\
+\left(1-\sum_{l m} \frac{1}{e^{\tilde{G}}} p^{* \delta_{\alpha}}(\tilde{G} ; l m)-\frac{1}{2 e^{\tilde{G}}}\right) \delta_{\alpha} v_{k(\tilde{G})}^{* \delta_{\alpha}}(\tilde{G}),
\end{array}
$$

where summations are over the set $\{l m \mid l m$ is $\tilde{G}$-efficient, with $l, m \neq k(\tilde{G})\}$. By an argument analogous to Step $1, v_{k(\tilde{G})}^{*}(\tilde{G} \ominus\{l, m\}) \geq 1 / 2$ for all $l m$. Taking the limit $\alpha \rightarrow \infty$, we obtain that

$$
v_{k(\tilde{G})}^{*}(\tilde{G}) \geq \pi^{\prime} \frac{1}{2}+\frac{1}{2 e^{\tilde{G}}}\left(1-v_{j(\tilde{G})}^{*}(\tilde{G})\right)+\left(1-\pi^{\prime}-\frac{1}{2 e^{\tilde{G}}}\right) v_{k(\tilde{G})}^{*}(\tilde{G}),
$$

where $\pi^{\prime}=\sum_{l m} \frac{1}{e^{\tilde{G}}} p^{*}(\tilde{G} ; \operatorname{lm}) \geq \frac{1}{e^{\tilde{G}}} p^{*}(\tilde{G} ; i(\tilde{G}) h(\tilde{G}))>0$. We can rewrite the inequality above as follows,

$$
\left(\pi^{\prime}+\frac{1}{2 e^{\tilde{G}}}\right) v_{k(\tilde{G})}^{*}(\tilde{G}) \geq \pi^{\prime} \frac{1}{2}+\frac{1}{2 e^{\tilde{G}}}\left(1-v_{j(\tilde{G})}^{*}(\tilde{G})\right)
$$

Since $v_{k(\tilde{G})}^{*}(\tilde{G})<1 / 2$, it must be that $v_{k(\tilde{G})}^{*}(\tilde{G})>1-v_{j(\tilde{G})}^{*}(\tilde{G})$, or equivalently $v_{j(\tilde{G})}^{*}(\tilde{G})+$ $v_{k(\tilde{G})}^{*}(\tilde{G})>1$.

Similarly, if $v_{h(\tilde{G})}^{*}(\tilde{G})<1 / 2$ then $v_{h(\tilde{G})}^{*}(\tilde{G})>1-v_{i(\tilde{G})}^{*}(\tilde{G})$. Since $v_{i(\tilde{G})}^{*}(\tilde{G}) \geq 1 / 2$, if $v_{h(\tilde{G})}^{*}(\tilde{G}) \geq 1 / 2$ then $v_{i(\tilde{G})}^{*}(\tilde{G})+v_{h(\tilde{G})}^{*}(\tilde{G}) \geq 1$. We established that $v_{i(\tilde{G})}^{*}(\tilde{G})+v_{h(\tilde{G})}^{*}(\tilde{G}) \geq 1$.

Consider the efficient match $\tilde{M}$ of $\tilde{G}$ that includes the links $i(\tilde{G}) h(\tilde{G})$ and $j(\tilde{G}) k(\tilde{G})$. The arguments above show that $v_{l}^{*}(\tilde{G})+v_{m}^{*}(\tilde{G}) \geq 1$ for all $l m \in \tilde{M}$, with strict inequality for $l m=$ $j(\tilde{G}) k(\tilde{G})$. Adding up these inequalities across all links in $\tilde{M}$ we obtain that $\sum_{l m \in \tilde{M}}\left(v_{l}^{*}(\tilde{G})+\right.$ $\left.v_{m}^{*}(\tilde{G})\right)>\mu(\tilde{G})$. This leads to a contradiction with the production technology constraint.

The series of contradictions above completes the proof as outlined in the first paragraph. 
Lemma 8. Suppose that $i \notin U(\tilde{G})$ and $v_{i}^{*}(\tilde{G})=1 / 2$. Then $v_{g}^{*}(\tilde{G}) \geq 1 / 2$ for every $g$ such that ig is a $\tilde{G}$-efficient link. If additionally $\tilde{\tilde{G}}$ is a subnetwork induced with positive limit probability under $\bar{\sigma}^{* \delta_{\alpha}}(\tilde{G})$ as $\alpha \rightarrow \infty$ and $i \in \tilde{\tilde{G}}$ then $v_{i}^{*}(\tilde{\tilde{G}})=1 / 2$.

Proof of Lemma 8. To prove the first part of the lemma, suppose that $i \notin U(\tilde{G})$ with $v_{i}^{*}(\tilde{G})=$ $1 / 2$, and $i g$ is a $\tilde{G}$-efficient link. Assume first that $\tilde{G}$ is a non-trivial perfect network. Then $g \notin U(\tilde{G})$ since $g$ is not isolated in $\tilde{G}$. Thus $v_{g}^{*}(\tilde{G}) \geq 1 / 2$ by the first part of Proposition 3 . Now assume that $\tilde{G}$ is not a non-trivial perfect network. A deviation by $i$ from $\bar{\sigma}_{i}^{* \delta_{\alpha}}(\tilde{G})$ to behavior that avoids any initial agreement except offering (slightly more than) $\delta_{\alpha} v_{g}^{* \delta_{\alpha}}(\tilde{G})$ to $g$ yields the following equilibrium requirement

$$
\begin{array}{r}
v_{i}^{* \delta_{\alpha}}(\tilde{G}) \geq \sum_{l m} \frac{1}{e^{\tilde{G}}} p^{* \delta_{\alpha}}(\tilde{G} ; l m) \delta_{\alpha} v_{i}^{* \delta_{\alpha}}(\tilde{G} \ominus\{l, m\})+\frac{1}{2 e^{\tilde{G}}}\left(1-\delta_{\alpha} v_{g}^{* \delta_{\alpha}}(\tilde{G})\right) \\
+\left(1-\sum_{l m} \frac{1}{e^{\tilde{G}}} p^{* \delta_{\alpha}}(\tilde{G} ; l m)-\frac{1}{2 e^{\tilde{G}}}\right) \delta_{\alpha} v_{i}^{* \delta_{\alpha}}(\tilde{G}),
\end{array}
$$

where summations are over the set $\{\operatorname{lm} \mid \operatorname{lm}$ is $\tilde{G}$-efficient, with $l, m \neq i\}$. For all $\tilde{G}$-efficient links $l m$ with $l, m \neq i$, we have that $i \notin U(\tilde{G} \ominus\{l, m\})$ since $i \notin U(\tilde{G})$. Thus $v_{i}^{*}(\tilde{G} \ominus\{l, m\}) \geq$ $1 / 2$ by the first part of Proposition 3. As $v_{i}^{*}(\tilde{G})=1 / 2$ and $v_{i}^{*}(\tilde{G} \ominus\{l, m\}) \geq 1 / 2$ for all $\tilde{G}$-efficient links $l m$, taking the limit $\alpha \rightarrow \infty$ we obtain that $v_{g}^{*}(\tilde{G}) \geq 1 / 2$.

For the second part of the lemma, it suffices to show that for any $G^{\prime} \in \mathcal{G}$, if $i \notin U\left(G^{\prime}\right)$ and $v_{i}^{*}\left(G^{\prime}\right)=1 / 2$ then $v_{i}^{*}\left(G^{\prime} \ominus\{l, m\}\right)=1 / 2$ for all $G^{\prime}$-efficient links $l m$ with $p^{*}\left(G^{\prime} ; l m\right)>0$. Fix such a $G^{\prime}$. For all $G^{\prime}$-efficient links $l m$, we have that $i \notin U\left(G^{\prime} \ominus\{l, m\}\right)$ since $i \notin U\left(G^{\prime}\right)$. Thus $v_{i}^{*}\left(G^{\prime} \ominus\{l, m\}\right) \geq 1 / 2$ by the first part of Proposition 3. If $G^{\prime}$ is a non-trivial perfect network, then for any $G^{\prime}$-efficient link $l m, \hat{U}\left(G^{\prime} \ominus\{l, m\}\right)=\emptyset$, hence $v_{i}^{*}\left(G^{\prime} \ominus\{l, m\}\right)=1 / 2$ by the second part of Proposition 3. If $G^{\prime}$ is a not non-trivial perfect network then a deviation by $i$ from $\bar{\sigma}_{i}^{* \delta_{\alpha}}\left(G^{\prime}\right)$ to behavior that avoids any initial agreement yields the following equilibrium requirement

$$
v_{i}^{* \delta_{\alpha}}\left(G^{\prime}\right) \geq \sum_{l m} \frac{1}{e^{G^{\prime}}} p^{* \delta_{\alpha}}\left(G^{\prime} ; l m\right) \delta_{\alpha} v_{i}^{* \delta_{\alpha}}\left(G^{\prime} \ominus\{l, m\}\right)+\left(1-\sum_{l m} \frac{1}{e^{G^{\prime}}} p^{* \delta_{\alpha}}\left(G^{\prime} ; l m\right)\right) \delta_{\alpha} v_{i}^{* \delta_{\alpha}}\left(G^{\prime}\right) .
$$

As $v_{i}^{*}\left(G^{\prime}\right)=1 / 2$ and $v_{i}^{*}\left(G^{\prime} \ominus\{l, m\}\right) \geq 1 / 2$ for all $G^{\prime}$-efficient links $l m$, taking the limit $\alpha \rightarrow$ $\infty$ we obtain that $v_{i}^{*}\left(G^{\prime} \ominus\{l, m\}\right)=1 / 2$ for all $G^{\prime}$-efficient links $l m$ with $p^{*}\left(G^{\prime} ; l m\right)>0$. 
Proof of Lemma 2. We construct the sequence iteratively. Suppose that we constructed $l_{1} m_{1}, \ldots, l_{s-1} m_{s-1}$, and that the goal has not been attained by step $s-1$, that is, $\hat{U}\left(\tilde{G}_{s}\right) \neq \emptyset$. Clearly, $i j$ is $\tilde{G}_{s}$-inefficient, thus $i, j \notin U\left(\tilde{G}_{s}\right)$. The definitions below identify the next link in the sequence, $l_{s} m_{s}$.

As $i$ and $j$ are always efficiently matched in $\tilde{G}$ with $v_{i}^{*}(\tilde{G})=v_{j}^{*}(\tilde{G})=1 / 2$, and $\tilde{G}_{s}$ is induced under $\bar{\sigma}^{* \delta_{\alpha}}(\tilde{G})$ with limit probability of at least $\left(e^{\tilde{G}}\right)^{-e^{\tilde{G}}}$ as $\alpha \rightarrow \infty$, Lemma 8 implies that $v_{i}^{*}\left(\tilde{G}_{s}\right)=v_{j}^{*}\left(\tilde{G}_{s}\right)=1 / 2$, and $v_{k}^{*}\left(\tilde{G}_{s}\right) \geq 1 / 2$ for all $k$ connected by $\tilde{G}_{s}$-efficient links to $i$ or $j$. Hence,

$$
v_{l}^{*}\left(\tilde{G}_{s}\right)+v_{m}^{*}\left(\tilde{G}_{s}\right) \geq 1 \text { for all } \tilde{G}_{s} \text {-efficient } l m \text { with }\{i, j\} \cap\{l, m\} \neq \emptyset .
$$

Suppose that there is no $\tilde{G}_{s}$-efficent link $l m$ in $\tilde{G}_{s} \ominus\{i, j\}$ with $p^{*}\left(\tilde{G}_{s} ; l m\right)=1$. Then

$$
v_{l}^{*}\left(\tilde{G}_{s}\right)+v_{m}^{*}\left(\tilde{G}_{s}\right) \geq 1 \text { for all } \tilde{G}_{s} \text {-efficient } l m \text { with }\{i, j\} \cap\{l, m\}=\emptyset .
$$

Thus all hypotheses of Lemma 7 are satisfied by $\tilde{G}_{s}$. Then there exist $l, m \in U\left(\tilde{G}_{s}\right) \cup$ $\left\{g \mid v_{g}^{*}\left(\tilde{G}_{s}\right)=1\right\}$ such that $p^{*}\left(\tilde{G}_{s} ; l m\right)=1$. As argued above, $i, j \notin U\left(\tilde{G}_{s}\right)$ and $v_{i}^{*}\left(\tilde{G}_{s}\right)=$ $v_{j}^{*}\left(\tilde{G}_{s}\right)=1 / 2<1$, hence $\{i, j\} \cap\{l, m\}=\emptyset$, leading to a contradiction. Therefore, there exists a $\tilde{G}_{s^{-}}$efficent link $l_{s} m_{s}$ in $\tilde{G}_{s} \ominus\{i, j\}$ such that $p^{*}\left(\tilde{G}_{s} ; l_{s} m_{s}\right)=1$. The link $l_{s} m_{s}$ is added to the sequence.

Proof of Lemma 3. The construction proceeds iteratively as in Lemma 2. Suppose that we constructed $l_{1} m_{1}, \ldots, l_{s-1} m_{s-1}$, and that the goal has not been attained by step $s-1$, that is, $j \notin\left\{i\left(\tilde{G}_{s}\right) h\left(\tilde{G}_{s}\right), j\left(\tilde{G}_{s}\right) k\left(\tilde{G}_{s}\right)\right\}$. The definitions below identify the next link in the sequence, $l_{s} m_{s}$.

Note that $\tilde{G}$ is a non-trivial perfect network because $\hat{U}(\tilde{G})=\emptyset$ and ij is $\tilde{G}$-inefficient. Then by construction, $\hat{U}\left(\tilde{G}_{s}\right)=\emptyset$ and $i j$ is $\tilde{G}_{s}$-inefficient, hence $\tilde{G}_{s}$ is also non-trivial perfect. Since $i$ can only belong to at most one of the sets $\left\{h\left(\tilde{G}_{s}\right), i\left(\tilde{G}_{s}\right)\right\}$ and $\left\{k\left(\tilde{G}_{s}\right), j\left(\tilde{G}_{s}\right)\right\}$, there is an ordered pair $\left(l_{s}, m_{s}\right)$ in the set $\left\{\left(h\left(\tilde{G}_{s}\right), i\left(\tilde{G}_{s}\right)\right),\left(k\left(\tilde{G}_{s}\right), j\left(\tilde{G}_{s}\right)\right)\right\}$ with $l_{s}, m_{s} \neq i$. As $j \notin\left\{h\left(\tilde{G}_{s}\right), i\left(\tilde{G}_{s}\right), j\left(\tilde{G}_{s}\right), k\left(\tilde{G}_{s}\right)\right\}$, we also have that $l_{s}, m_{s} \neq j$. The link $l_{s} m_{s}$ is added to the sequence.

Proof of Lemma 4. Let $h$ be $i$ 's match in an arbitrary efficient match of $\tilde{G}$ that includes the link $j k$. The construction proceeds iteratively as in Lemma 2. Suppose that we constructed $l_{1} m_{1}, \ldots, l_{s-1} m_{s-1}$, and that the goal has not been attained by step $s-1$, that is, the set 
of players in $\tilde{G}_{s} \ominus\{h, i, j, k\}$ is not $\tilde{G}_{s}$-efficiently closed. The definitions below identify the next link in the sequence, $l_{s} m_{s}$.

By construction, $i j$ is $\tilde{G}_{s}$-inefficient, thus $i$ and $j$ are always efficiently matched in $\tilde{G}_{s}$. Suppose that there are no players in $\tilde{G}_{s} \ominus\{h, i, j, k\}$ that have $\tilde{G}_{s}$-efficient links to either $i$ or $j$. Then each of $i$ and $j$ can only have $\tilde{G}_{s}$-efficient links to $h$ or $k$ ( $i j$ is $\tilde{G}_{s}$-inefficient). Since $i$ and $j$ are always efficiently matched in $\tilde{G}_{s}$, it must be that in every efficient match of $\tilde{G}_{s}$ each of the players $h$ and $k$ is matched to one of the players $i$ and $j$. Thus $\{h, i, j, k\}$ is $\tilde{G}_{s}$-efficiently closed, which leads to a contradiction with the assumption that the set of players in $\tilde{G}_{s} \ominus\{h, i, j, k\}$ is not $\tilde{G}_{s}$-efficiently closed.

We established that there is a player $m_{s}$ in $\tilde{G}_{s} \ominus\{h, i, j, k\}$ that has a $\tilde{G}_{s}$-efficient link to either $i$ or $j$. Let $l_{s}$ be $m_{s}$ 's match in the efficient match of $\tilde{G}$ that includes the links $i h$ and $j k\left(m_{s}\right.$ is not isolated in $\tilde{G}$ as it is connected to $i$ or $j$, thus it is always efficiently matched in $\tilde{G})$. The link $l_{s} m_{s}$ is added to the sequence.

Proof of Lemma 5. The construction proceeds iteratively as in Lemma 2. Suppose that we constructed $l_{1} m_{1}, \ldots, l_{s-1} m_{s-1}$, and that the goal has not been attained by step $s-1$, that is, there exist non-isolated players in $\tilde{G}_{s}$ different from $h, i, j, k$. The definitions below identify the next link in the sequence, $l_{s} m_{s}$.

By construction, the set of players in $\tilde{G}_{s} \ominus\{h, i, j, k\}$ is $\tilde{G}_{s}$-efficiently closed. Then the assumption that there exist non-isolated players in $\tilde{G}_{s}$ different from $h, i, j, k$ implies that $\tilde{G}_{s} \ominus\{h, i, j, k\}$ has a non-empty set of links (every non-isolated player in $\tilde{G}_{s}$ different from $h, i, j, k$ has at least one $\tilde{G}_{s}$-efficient link; such links need to belong to $\tilde{G}_{s} \ominus\{h, i, j, k\}$ because the set of players in $\tilde{G}_{s} \ominus\{h, i, j, k\}$ is $\tilde{G}_{s}$-efficiently closed). Then by Lemma 1 there is a link $l_{s} m_{s}$ in $\tilde{G}_{s} \ominus\{h, i, j, k\}$ such that $p^{*}\left(\tilde{G}_{s} ; l_{s} m_{s}\right)=1$. The link $l_{s} m_{s}$ is added to the sequence.

\section{Appendix C. Proofs for Section 4}

The system of equations solved by $v^{* \delta}\left(G_{\text {line } 4}\right)$ for low $\delta$. For low $\delta$ there is a unique MPE of $\Gamma^{\delta}\left(G_{\text {line }} 4\right)$. In any subgame, every match ends in agreement. By the proof of Proposition 1 
and by symmetry, the equilibrium payoffs solve the following system,

$$
\begin{aligned}
& v_{1}=\frac{1}{3} \frac{1}{2}\left(\left(1-\delta v_{2}\right)+\delta v_{1}\right)+\frac{1}{3} 0+\frac{1}{3} \delta / 2 \\
& v_{2}=\frac{1}{3} \frac{1}{2}\left(\delta v_{2}+\left(1-\delta v_{1}\right)\right)+\frac{1}{3} \frac{1}{2}\left(\left(1-\delta v_{3}\right)+\delta v_{2}\right)+\frac{1}{3} \delta / 2 \\
& v_{3}=v_{2}, v_{4}=v_{1} .
\end{aligned}
$$

The unique solution is given by

$$
v_{1}^{* \delta}\left(G_{\text {line } 4}\right)=v_{4}^{* \delta}\left(G_{\text {line } 4}\right)=\frac{6+3 \delta-2 \delta^{2}}{12(3-\delta)}, v_{2}^{* \delta}\left(G_{\text {line } 4}\right)=v_{3}^{* \delta}\left(G_{\text {line } 4}\right)=\frac{12+3 \delta-2 \delta^{2}}{12(3-\delta)} .
$$

There is an MPE with payoffs as above only if $2 \delta \frac{12+3 \delta-2 \delta^{2}}{12(3-\delta)} \leq 1$, so that $\delta\left(v_{2}^{* \delta}\left(G_{\text {line } 4}\right)+\right.$ $\left.v_{3}^{* \delta}\left(G_{\text {line } 4}\right)\right) \leq 1$. Equivalently, $\delta \leq \underline{\delta} \approx .945$, where $\underline{\delta}$ is the unique root in the interval $[0,1]$ of the polynomial $18-18 x-3 x^{2}+2 x^{3}$.

The system of equations solved by $v^{* \delta}\left(G_{\text {line } 4}\right)$ for $\delta>\underline{\delta}$. For $\delta>\underline{\delta}$, there exists an MPE of $\Gamma^{\delta}\left(G_{\text {line } 4}\right)$ in which players 2 and 3 reach agreement with some probability $p \in(0,1)$ conditional on their link being selected for bargaining. ${ }^{26}$ As in the proof of Proposition 1, we need the equilibrium payoffs of players 2 and 3 to satisfy $\delta\left(v_{2}^{* \delta}\left(G_{\text {line } 4}\right)+v_{3}^{* \delta}\left(G_{\text {line } 4}\right)\right)=1$. By symmetry, the equilibrium payoffs solve the following system,

$$
\begin{aligned}
v_{1} & =\frac{1}{3} \frac{1}{2}\left(\left(1-\delta v_{2}\right)+\delta v_{1}\right)+\frac{1}{3}(1-p) \delta v_{1}+\frac{1}{3} \delta / 2 \\
v_{2} & =\frac{1}{3} \frac{1}{2}\left(\delta v_{2}+\left(1-\delta v_{1}\right)\right)+\frac{1}{3} \delta v_{2}+\frac{1}{3} \delta / 2 \\
\delta\left(v_{2}+v_{3}\right) & =1, v_{3}=v_{2}, v_{4}=v_{1} .
\end{aligned}
$$

The unique solution is given by

$$
\begin{aligned}
v_{1}^{* \delta}\left(G_{\text {line } 4}\right)=v_{4}^{* \delta}\left(G_{\text {line } 4}\right)=\frac{-6+5 \delta+2 \delta^{2}}{2 \delta^{2}}, v_{2}^{* \delta}\left(G_{\text {line } 4}\right)=v_{3}^{* \delta}\left(G_{\text {line } 4}\right)=\frac{1}{2 \delta} \\
p^{* \delta}=\frac{2\left(9-12 \delta+\delta^{2}+2 \delta^{3}\right)}{\delta\left(-6+5 \delta+2 \delta^{2}\right)} .
\end{aligned}
$$

\footnotetext{
${ }^{26}$ The probabilities that 2 accepts an offer from 3 and that 3 accepts an offer from 2 are not pinned down by the MPE requirements. Only the average $p$ of the two conditional probabilities is relevant for MPE payoff computation. There exist multiple MPEs, all payoff equivalent, as outlined in footnote 24.
} 
Proof of Proposition 4. We first show that the system 4.1 has a unique solution $v^{\delta, H}$, and that $v^{\delta, H}$ belongs to $[0,1]^{n}$. Note that a vector is a solution to 4.1 if and only if it is a fixed point of the function $h: \mathbb{R}^{n} \rightarrow \mathbb{R}^{n}$ defined by

$$
h_{k}(v)=\frac{2\left(e^{G}-e^{H}\right)+e_{k}^{H}}{2 e^{G}} \delta v_{k}+\frac{1}{2 e^{G}} \sum_{\{i \mid k i \in H\}}\left(1-\delta v_{i}\right)+\frac{1}{e^{G}} \sum_{\{i j \mid i j \in H \ominus\{k\}\}} \delta v_{k}^{* \delta}(G \ominus\{i, j\}) .
$$

It is easily checked that $h$ is a contraction with respect to the sup norm on $\mathbb{R}^{n}$, mapping $[0,1]^{n}$ into itself, hence it has a unique fixed point, denoted $v^{\delta, H}$, which belongs to $[0,1]^{n}$. Therefore, for all $\delta \in(0,1), v^{\delta, H}$ is the unique solution to 4.1. In particular, the linear system 4.1 is non-singular.

If $G$ has more than one link, then a more convoluted contraction argument establishes that the linear system 4.2 is non-singular, and the unique solution $v^{H}$ belongs to $[0,1]^{n} .^{27}$

We next extablish that $\lim _{\delta \rightarrow 1} v_{l}^{\delta, H}=v_{l}^{H}$ for all $l \in N$. Consider the linear system 4.1. All entries in the coefficient matrix are linear functions of $\delta$, and the entries in the augmented matrix are polynomial functions of $\delta$ and $v_{k}^{* \delta}(G \ominus\{i, j\})$ for triplets of players $(i, j, k)$ with $i j \in H$ and $k \neq i, j$ (henceforth, $i j k$ refers to any such triplet). Then $v_{l}^{\delta, H}$ is computed by Cramer's rule, as the ratio of two determinants,

$$
v_{l}^{\delta, H}=P_{l}^{H}\left(\delta,\left(v_{k}^{* \delta}(G \ominus\{i, j\})\right)_{i j k}\right) / Q^{H}(\delta),
$$

where $P_{l}^{H}$ is a polynomial in multiple variables and $Q^{H}$ is a polynomial in one variable. $Q^{H}(\delta) \neq 0$ for all $\delta \in(0,1)$ since the corresponding linear systems 4.1 are non-singular. We can also compute $v_{l}^{H}$ by Cramer's rule,

$$
v_{l}^{H}=P_{l}^{H}\left(1,\left(v_{k}^{*}(G \ominus\{i, j\})\right)_{i j k}\right) / Q^{H}(1) .
$$

Note that $Q^{H}(1) \neq 0$ since the linear system 4.2 is non-singular. Because $P_{l}^{H}$ and $Q^{H}$ are polynomial functions, they are continuous in their arguments, hence $\lim _{\delta \rightarrow 1} P_{l}^{H}\left(\delta,\left(v_{k}^{* \delta}(G \ominus\right.\right.$

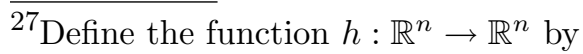

$$
h_{k}(v)=\frac{2\left(e^{G}-e^{H}\right)+e_{k}^{H}}{2 e^{G}} v_{k}+\frac{1}{2 e^{G}} \sum_{\{i \mid k i \in H\}}\left(1-v_{i}\right)+\frac{1}{e^{G}} \sum_{\{i j \mid i j \in H \ominus\{k\}\}} v_{k}^{*}(G \ominus\{i, j\}) .
$$

If $G$ has more than one link then one can prove that $h \circ h$ is a contraction with respect to the sup norm on $\mathbb{R}^{n}$, and maps $[0,1]^{n}$ into itself. Hence $h \circ h$ has a unique fixed point $v^{H}$, which belongs to $[0,1]^{n}$. But if $v^{H}$ is a fixed point of $h \circ h$, so is $h\left(v^{H}\right)$. Then the fact that $v^{H}$ is the unique fixed point of $h \circ h$ implies that $h\left(v^{H}\right)=v^{H}$, i.e., $v^{H}$ is a fixed point of $h$. However, $h$ cannot have any fixed points different from $v^{H}$, since any fixed point of $h$ is a fixed point of $h \circ h$. Hence $v^{H}$ is the unique fixed point of $h$. 


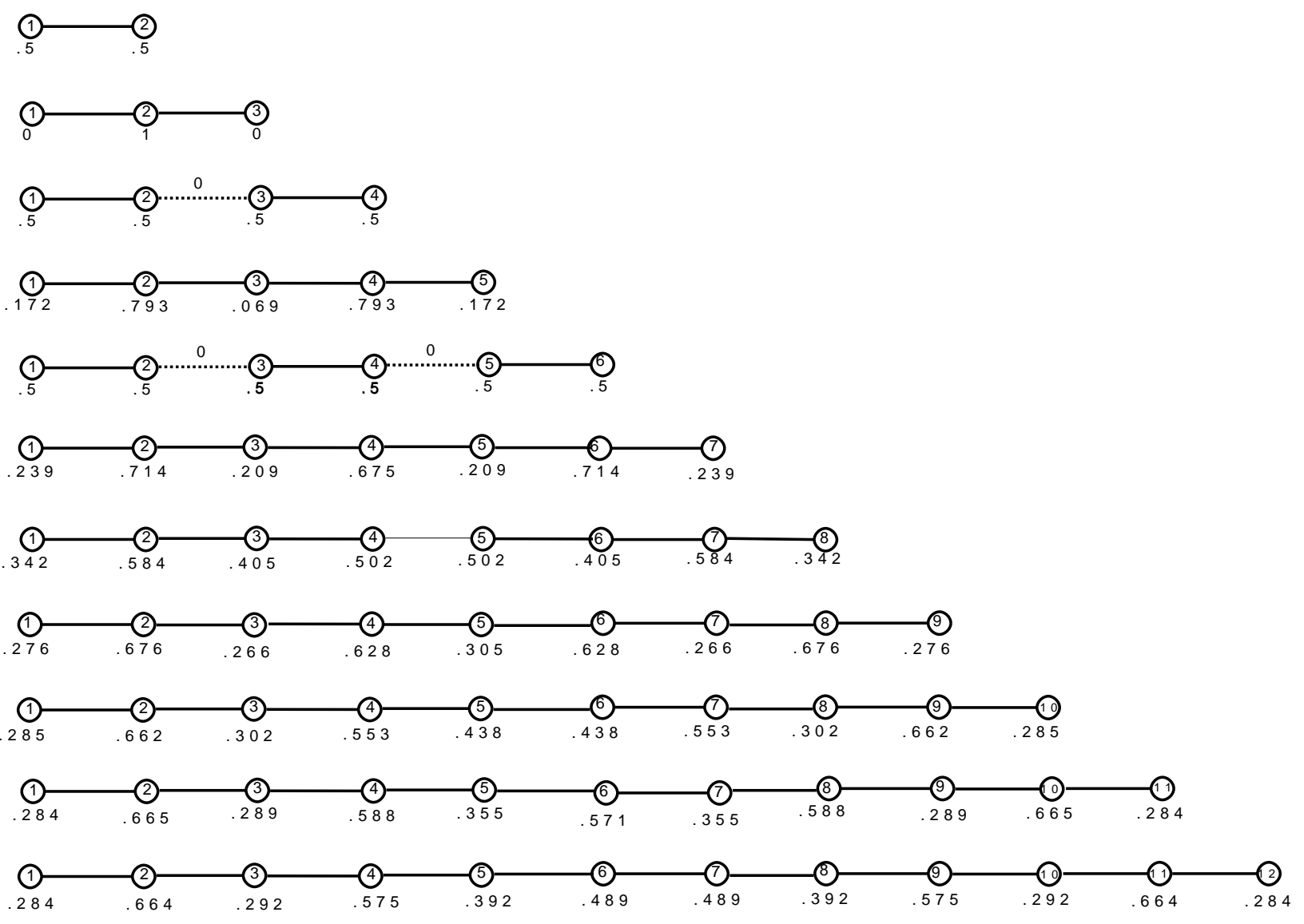

FiguRE 5. Limit MPE payoffs and initial agreements for the bargaining games on the line of $2,3, \ldots, 12$ networks.

$\left.\{i, j\}))_{i j k}\right)=P_{l}^{H}\left(1,\left(v_{k}^{*}(G \ominus\{i, j\})\right)_{i j k}\right)$ and $\lim _{\delta \rightarrow 1} Q^{H}(\delta)=Q^{H}(1)$. Therefore, $\lim _{\delta \rightarrow 1} v_{l}^{\delta, H}=$ $v_{l}^{H}$.

Suppose $v^{H}$ satisfies $v_{i}^{H}+v_{j}^{H}<1$ for $i j \in H$ and $v_{i}^{H}+v_{j}^{H}>1$ for $i j \in G, i j \notin H$. Since $\lim _{\delta \rightarrow 1} v^{\delta, H}=v^{H}$ it follows that there exists $\underline{\delta}$ such that for every $\delta \in(\underline{\delta}, 1), v^{\delta, H}$ satisfies $v_{i}^{\delta, H}+v_{j}^{\delta, H}<1$ for $i j \in H$ and $v_{i}^{\delta, H}+v_{j}^{\delta, H}>1$ for $i j \in G, i j \notin H$. For $\delta \in(\underline{\delta}, 1)$, since $v^{\delta, H}$ solves 4.1, it follows that $v^{\delta, H}$ is a fixed point of the correpsondence $f$ defined using $\left(v_{k}^{* \delta}(G \ominus\{i, j\})\right)_{i j k}$ as in Appendix A. Lemma 6 implies that, for every $\delta \in(\underline{\delta}, 1), \Gamma^{\delta}(G)$ has an MPE with payoffs $v^{\delta, H}$.

The limit linear system 4.2 corresponding to $\Gamma^{\delta}\left(G_{\text {sq+line } 3}\right)$ and the subnetwork $H$. In each equation the terms correspond in order to the selection for bargaining of the links $(k, k+1)$ for 
$k=1,2, \ldots, 6$, followed by the link $(1,4)$,

$$
\begin{aligned}
& v_{1}=\frac{1}{7} \frac{1}{2}\left(v_{1}+1-v_{2}\right)+\frac{1}{7} 5 / 29+\frac{1}{7} 1 / 2+\frac{1}{7} 0+\frac{1}{7} 1 / 2+\frac{1}{7} 0+\frac{1}{7} v_{1} \\
& v_{2}=\frac{1}{7} \frac{1}{2}\left(v_{2}+1-v_{1}\right)+\frac{1}{7} \frac{1}{2}\left(v_{2}+1-v_{3}\right)+\frac{1}{7} 1 / 2+\frac{1}{7} 1+\frac{1}{7} 1 / 2+\frac{1}{7} 1+\frac{1}{7} v_{2} \\
& v_{3}=\frac{1}{7} 5 / 29+\frac{1}{7} \frac{1}{2}\left(v_{3}+1-v_{2}\right)+\frac{1}{7} \frac{1}{2}\left(v_{3}+1-v_{4}\right)+\frac{1}{7} 0+\frac{1}{7} 1 / 2+\frac{1}{7} 0+\frac{1}{7} v_{3} \\
& v_{4}=\frac{1}{7} 23 / 29+\frac{1}{7} 23 / 29+\frac{1}{7} \frac{1}{2}\left(v_{4}+1-v_{3}\right)+\frac{1}{7} \frac{1}{2}\left(v_{4}+1-v_{5}\right)+\frac{1}{7} 1 / 2+\frac{1}{7} 1+\frac{1}{7} v_{4} \\
& v_{5}=\frac{1}{7} 2 / 29+\frac{1}{7} 2 / 29+\frac{1}{7} 0+\frac{1}{7} \frac{1}{2}\left(v_{5}+1-v_{4}\right)+\frac{1}{7} \frac{1}{2}\left(v_{5}+1-v_{6}\right)+\frac{1}{7} 0+\frac{1}{7} v_{5} \\
& v_{6}=\frac{1}{7} 23 / 29+\frac{1}{7} 23 / 29+\frac{1}{7} 1+\frac{1}{7} 1 / 2+\frac{1}{7} \frac{1}{2}\left(v_{6}+1-v_{5}\right)+\frac{1}{7} \frac{1}{2}\left(v_{6}+1-v_{7}\right)+\frac{1}{7} v_{6} \\
& v_{7}=\frac{1}{7} 5 / 29+\frac{1}{7} 5 / 29+\frac{1}{7} 0+\frac{1}{7} 1 / 2+\frac{1}{7} 0+\frac{1}{7} \frac{1}{2}\left(v_{7}+1-v_{6}\right)+\frac{1}{7} v_{7} .
\end{aligned}
$$

The unique solution is $v^{H}$ with $v_{1}^{H} \approx 0.235, v_{2}^{H} \approx 0.759, v_{3}^{H} \approx 0.179, v_{4}^{H} \approx 0.792, v_{5}^{H} \approx$ $0.069, v_{6}^{H} \approx 0.793, v_{7}^{H} \approx 0.172 .^{28}$

\section{REFERENCES}

1. Binmore, K.G. (1987) Perfect-Equilibria in Bargaining Models, in K. Binmore and P. Dasgupta (eds.), The Economics of Bargaining, Basil Blackwell, Oxford.

2. Binmore, K.G. and M.J. Herrero (1988) Matching and Bargaining in Dynamic Markets, Rev. Econ. Stud., 55, 17-31.

3. Bogomolnaia, A. and H. Moulin (2004) Random Matching Under Dichotomous Preferences, Econometrica, 72, 257-279.

4. Calvo-Armengol, A. (2001) Bargaining power in communication networks, Math. Social Sci., 41, 69-88.

5. Calvo-Armengol, A. (2003) Stable and efficient bargaining networks, Rev. Econ. Design, 7, 411-428.

6. Corominas-Bosch, M. (2004) Bargaining in a network of buyers and sellers, J. Econ. Theory, 115, 35-77.

7. Demange, G., D. Gale and M. Sotomayor (1986) Multi-Item Auctions, J. Polit. Economy, 94, 863-872.

8. Dutta, P. K. (1995) A Folk theorem for stochastic games, J. Econ. Theory, 66, 1-32.

9. Fudenberg, D. and J. Tirole (1991) Game Theory, MIT Press.

10. Gale, D. (1987) Limit Theorems for Markets with Sequential Bargaining, J. Econ. Theory, 43, 20-54.

11. Jackson, M.O. (2008) Social and Economic Networks, Princeton University Press.

12. Kranton, R. and D. Minehart (2001) A Theory of Buyer-Seller Networks, Amer. Econ. Rev., 91, 485-508.

\footnotetext{
${ }^{28}$ The exact solution involves irreducible fractions with 8-digit denominators.
} 
13. Lovasz, L. and M.D. Plummer (1986) Matching Theory, North-Holland, Amsterdam.

14. Manea, M. (2008) Bargaining in Stationary Networks, mimeo.

15. Maskin, M. and J. Tirole (2001) Markov Perfect Equilibrium, I. Observable Actions, J. Econ. Theory, 100, 191-219.

16. Mertens, J.-F. (2002) Stochastic games, in R.J. Aumann and S. Hart (eds.), Handbook of Game Theory with Economic Applications, Elsevier.

17. Polanski, A. (2007) Bilateral bargaining in networks, J. Econ. Theory, 134, 557-565.

18. Roth, A., T. Sonmez and U. Unver (2005) Pairwise Kidney Exchange, J. Econ. Theory, 125, 151-188.

19. Rubinstein, A. (1982) Perfect equilibria of a bargaining model, Econometrica, 50, 97-110.

20. Rubinstein, A. and U. Wolinsky (1985) Equilibrium in a Market with Sequential Bargaining, Econometrica, 53, 295-328.

21. Rubinstein, A. and U. Wolinsky (1990) Decentralized Trading, Strategic Behaviour and the Walrasian Outcome, Rev. Econ. Stud., 57, 1, 63-78.

22. Stahl, I. (1972) Bargaining Theory, Stockholm School of Economics, Stockholm.

23. Wen, Q. (2002) A Folk Theorem for Repeated Sequential Games, Rev. Econ. Stud., 69, 493-512. 